\title{
Acoustic Travel Time Perturbations due to an Internal Tide and Internal Wave Field in the Barents Sea
}

by

\author{
Douglas Scott Ray \\ B.S. Mechanical Engineering \\ United States Naval Academy (1980)
}

Submitted in partial fulfillment of the requirements for the degree of

OCEAN ENGINEER

at the

MASSACHUSETTS INSTITUTE OF TECHNOLOGY
and

WOODS HOLE OCEANOGRAPHIC INSTITUTION

August 1993

(c) Douglas Scott Ray, MCMXCIII. All rights reserved.

\begin{tabular}{c} 
MAPINE \\
BOLOEICAL \\
LABORATORY \\
\hline LIBRARY \\
\hline \begin{tabular}{c} 
WOODS HOLE, MASS. \\
W. H. O. 1. \\
\hline
\end{tabular} \\
\hline
\end{tabular}

The author hereby grants to MIT, WHOI and the U.S. Government permission to reproduce and to distribute copies of this thesis document in whole or in part.

Author

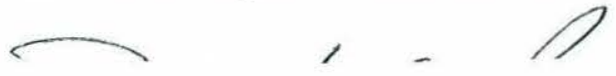

Department of Ocean EAgineering, Massachussetts Institute of Technology, and the Joint Program in Oceanography and Oceanographic Engineering, Massachussetts Institute of Technology/Woods Hole Oceanographic Institution August 6, 1993 Certified by $\ldots \ldots \ldots$ Dr. James F. Lynch
Associate Scientist, Woods Hotesis Supervisor

Certified by

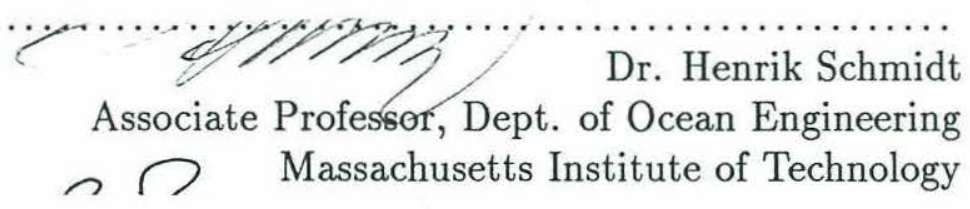

Accepted by

Dr. Arthur B. Baggeroer

Chairman, Joint Committee for Applied Ocean Science and Engineering Massachusetts Institute of Technology/Woods Hole Oceanographic Institution

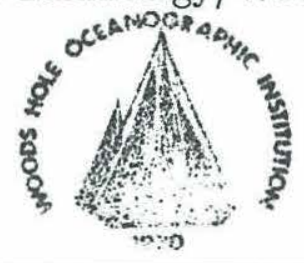




\title{
Acoustic Travel Time Perturbations due to an Internal \\ Tide and Internal Wave Field in the Barents Sea
}

\author{
by \\ Douglas Scott Ray
}

Submitted to the Department of Ocean Engineering, Massachussetts Institute of Technology, and the Joint Program in Oceanography and Oceanographic Engineering, Massachussetts Institute of Technology/Woods Hole Oceanographic Institution on August 6, 1993, in partial fulfillment of the

requirements for the degree of

OCEAN ENGINEER

\begin{abstract}
Travel time perturbations of adiabatic normal modes due to an internal tide and internal mode field in the Barents Sea are examined. A formalism for the travel time perturbation due to a change in sound speed is presented. Internal tide and internal wave amplitude spectra are calculated from Brancker temperature loggers which were deployed on moorings in the Barents Sea during the August 1992 Barents Sea Polar Front Experiment. In particular, the first three internal wave mode amplitudes are estimated from the four Brancker temperature loggers on the southwest mooring of the array. Modal perturbations in acoustic pulse travel time and the travel time covariance are calculated and compared for consistency to a simple ray model. These perturbations are small for the modal arrivals that the vertical acoustic array which was deployed is expected to resolve. The third internal wave mode has the largest impact on the acoustic arrivals, per unit amplitude, but the first internal wave mode dominates the scattering due to having a much larger amplitude overall.
\end{abstract}

Thesis Supervisor: Dr. James F. Lynch

Title: Associate Scientist

Woods Hole Oceanographic Institution 


\section{Acknowledgments}

I would like to thank my thesis advisor, Dr. James F. Lynch, for his guidance, support, and motivation. He provided encouragement when things went badly, direction when I strayed from the path, and patience when I stumbled and erred as I learned.

I would also like to thank Art Newhall and Rich Pawlowicz, who showed me the inner workings and hidden mechanisms of computers in general and UNIX in

particular. Without their continual help, I would have been able to accomplish only a fraction of what I did. I am also very much indebted to Guoliang Jin, who derived the internal wave scattering formulation in this thesis.

I am grateful for the association I enjoyed with my fellow students, who helped me in countless ways. In particular, Steve Bowen, Matt Johnson, Joe Bondaryk, John Buck, Matt Conti, Dennis Wojcik, and Fred Thwaites listened to my problems, answered my questions, and discussed the major and minor issues of the day with me, all of which served to maintain a sense of perspective and humor.

I wish to thank the United States Navy and the Oceanographer of the Navy for allowing me the opportunity to participate in the Joint Program, and especially Captain Ralph Schlichter, USN and Commander Gerald Harvey, USN, who supported me in coming here. I additionally wish to thank the Office of Naval Research (ONR Code 1125AR), who funded the Barents Sea Tomography Transmission Test, thereby giving me the data to work on.

Finally, I would like to thank my wife Terry for her unending love, support, patience and faith throughout this time. I would also like to thank my children, Melissa, Morgan, Stephanie, Daniel, and Kyle, whose love and very presence are a continual source of joy and wonderment. 


\section{To my wife, Terry and to my children, Melissa, Morgan, Stephanie, Daniel, and Kyle}




\section{Contents}

1 Introduction $\quad 11$

1.1 Motivation . . . . . . . . . . . . . . . . 11

1.2 Thesis Objectives ............................ 13

1.3 Thesis Content . . . . . . . . . . . . . . . . 13

2 Theory 14

2.1 Acoustic Tomography . . . . . . . . . . . . . . 14

2.2 Internal Motions in the Ocean . . . . . . . . . . . . 16

2.2 .1 Internal Tides . . . . . . . . . . . . . 16

2.2 .2 Internal Waves . . . . . . . . . . . . . 18

2.3 Adiabatic Acoustic Normal Modes . . . . . . . . . . . . . . 23

2.4 Sound speed perturbations due to Internal Waves . . . . . . . . 25

3 Barents Sea Polar Front Experiment 31

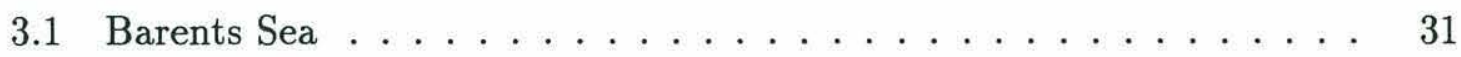

3.2 Experimental Objectives . . . . . . . . . . . . . 34

3.3 Data Acquisition ...................... 34

4 Temperature Field Data Analysis $\quad 38$

4.1 Horizontal Advection versus Internal Tides and Waves . . . . . . . 39

4.2 The Internal Tide and Internal Wave Spectrum . . . . . . . . . 43

4.3 Review of Assumptions . . . . . . . . . . . . . . . . 49

5 Travel Time Disturbance Predictions $5 \mathbf{5 2}$ 
5.1 Theoretical Results for the M2 Tide . . . . . . . . . . . . . 52

5.1 .1 Acoustic Ray Results . . . . . . . . . . . . . . . 53

5.1.2 Acoustic Normal Mode Results . . . . . . . . . . . 55

5.1.3 Comparison of Ray and Modal Results . . . . . . . . . . . 60

5.2 Acoustic Normal Mode Results for the Internal Tide and Internal Wave

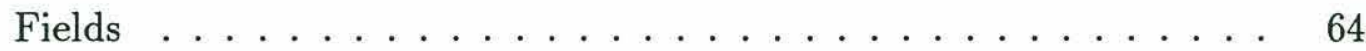

5.3 Movement of the Front . . . . . . . . . . . . . . . 67

6 Conclusions and Future Work $\quad 68$ 


\section{List of Figures}

2-1 Power Spectrum Density of Brancker temperature logger \#3663 located on NE array at a depth of $32.1 \mathrm{~m}$. Evident are the M2 tide, its higher harmonics, and the internal wave band. $N_{\max }$ at this depth is 215 cpd. The estimate has 4 degrees of freedom. . . . . . . . . .

3-1 Chart of the Barents Sea with major currents. The thick, solid line is the Barents Sea Polar Front. . . . . . . . . . . . . . . . . 32

3-2 Chart of the BSPF experiment. Triangles are array positions. Arrows denote the acoustic paths during the experiment. . . . . . . . . 35

3-3 CTD grid employed during BSPF experiment. Circles are CTD stations. The grid was fully covered three times. . . . . . . . . . . . 36

4-1 Temperature record from Brancker logger \# 3666 located on the SW mooring at a depth of $29.9 \mathrm{~m}$. Brancker temperature resolution is $0.1{ }^{\circ} \mathrm{C}$

4-2 (a) is a $9.9 \mathrm{~m}$ sinusoidal particle displacement about the logger depth (dashed line). (b) is a smoothed temperature profile at the SW mooring. The dashed line is the logger depth. (c) is the "distorted" temperature record which results from the sinusoidal particle displacement in a varying temperature gradient. The dashed line is the axis of zero

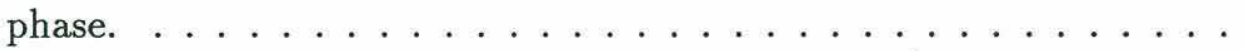

4-3 Three temperature profiles at the SW array taken over a span of 18 days. The temperature records show both a temperature change due to vertical displacement (caused by internal tides and waves) and due to horizontal advection (caused by the barotropic tide) . . . . . . . . 41 
4-4 $U$ and $V$ velocity, and Temperature records from Aanderaa \#9259 at the NE array at a depth of 82.1 meters. . . . . . . . . . . . . . . 42

4-5 Temperature contour map at NE mooring at a depth of $82.1 \mathrm{~m}$ using adjacent CTD stations. The current ellipse from Aanderaa \# 9259 shows the amount of horizontal advection during one tidal cycle. . .

4-6 Temperature and Salinity versus depth for the CTD casts taken at the NE array. . . . . . . . . . . . . . . . . . . . . 44

4-7 Temperature record for Brancker logger \#3663 at NE mooring at a depth of $32.1 \mathrm{~m} . \ldots \ldots \ldots \ldots \ldots$. . . . . . . . . . . . . . 45

4-8 Particle displacement $\zeta$ calculated for Brancker logger \# 3663 on NE array at a depth of $32.1 \mathrm{~m} . \ldots \ldots$. . . . . . . . . . . . 46

4-9 Power spectrum density for the particle displacement calculated for Brancker logger \# 3663 on the NE array at a depth of $32.1 \mathrm{~m}$. The estimate has four degrees of freedom. . . . . . . . . . . .

4-10 Amplitude spectrum for Brancker logger \# 3660 on SW array at a depth of $39.9 \mathrm{~m} . \ldots \ldots \ldots \ldots \ldots$. . . . . . . . . . . . . . . . 48

4-11 (a) is the least squares fit of four amplitudes measured at the SW array to a sum of the first four IW modes. (b) is the same calculation except that the two shallow Branckers are averaged together. In both figures, considerable noise is evident in the internal tide region, and the method is unreliable above about $27 \mathrm{cpd} \ldots \ldots \ldots \ldots$

5-1 Bathymetry of two segment range dependent model from NE array to SE array. Solid line is the depth of the model. Dashed line is the depth at each CTD station. Segment \#1 goes from 0-26.93km. . . . . . .

5-2 (a) Averaged sound speed profile for segment \# 1, and (b) Averaged sound speed profile for segment \# 2 . . . . . . . . . . . . 54

5-3 Travel time perturbation of eigenrays versus launch angle. . . . . . . 56

5-4 Smoothed buoyancy frequency $N$ profiles for (a) segment \# 1 and (b) segment \# $2 \ldots \ldots \ldots \ldots \ldots \ldots \ldots$ 
5-5 First three internal tide modes for (a) segment \# 1 and (b) segment $\# 2$ at $\omega=M 2 \ldots \ldots \ldots \ldots \ldots \ldots$

5-6 First three adiabatic normal modes for (a) segment \# 1 and (b) segment \# 2. . . . . . . . . . . . . . . . . . 58

5-7 Travel time perturbations at $M 2$ using first ten internal tide modes equally weighted with a maximum amplitude of $5.2 \mathrm{~m}$. Lines are acous-

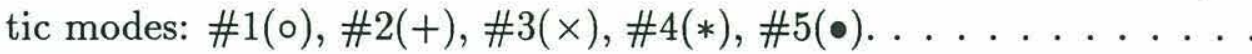

5-8 Travel time perturbations at $M 2$. The first 3 internal tide modes are shown and are weighted by $0.58,0.26$, and 0.16 , respectively. . . . . 61

5-9 Travel time perturbations at $M 2$ using the sum of the first 3 internal tide modes, weighted by $0.58,0.26$, and 0.16 . Shown for comparison (as a dashed line) is the contribution from the first internal tide mode at $M 2$ (Fig. 5-8) . . . . . . . . . . . . . . . .

5-10 RMS of the travel time perturbations at $M 2$ using the first three internal tide modes. . . . . . . . . . . . . . . . . . .

5-11 Travel time perturbations using the first three IW modes from $f$ to $N$. They are weighted by $0.58,0.26$, and 0.16 . Shown for comparison (as a dashed line) is the contribution from the first internal tide mode at $M 2$ (Fig. 5-8) . . . . . . . . . . . . . . . .

5-12 RMS of the travel time perturbations, using the first three IW modes, weighted by $0.58,0.26$, and 0.16 , and from $f$ to $N$. The dashed line is the rms of the travel time perturbation for the $M 2$ component. The dotted line is rms of the travel time perturbation for the across shelf case. For segment \#2 the dotted line lies directly over the dashed line. 


\section{List of Tables}

3.1 Water Mass Definitions . . . . . . . . . . . . . . 33

3.2 Tidal Amplitudes at Bear Island . . . . . . . . . . . . . . . . . . 34

3.3 Barents Sea Moorings . . . . . . . . . . . . . . 37

4.1 Horizontal Advection of Water due to Barotropic Tides . . . . . . . . 43

4.2 SW Mooring Particle Displacement Amplitudes $\zeta \ldots . . . . . . . .47$

5.1 Two-layer, range independent model . . . . . . . . . . . 55 


\section{Chapter 1}

\section{Introduction}

Tomography comes from the Greek word tomos meaning slice or section. Ocean acoustic tomography is the science of taking acoustic slices or sections of the ocean sound speed, temperature, and current fields. In this thesis we will deal with the application of tomographic techniques to the study of internal waves and internal tides.

\subsection{Motivation}

The ocean covers over two-thirds of the earth's surface. With its tremendous heat capacity and enormous currents, the ocean exerts a significant influence on the world's climate and meteorology. This influence is not well understood; in part due to the difficulty in observing the ocean volume, and also in part due to the ocean's mesoscale and inter-annual variability. Remote sensing using electromagnetic energy in the forms of light and radar, which has allowed man to peer billions of light-years into outer space, encounters in the ocean an opaque medium. The average depth of the ocean is $4000 \mathrm{~m}$, but blue-green light and radar can only penetrate to depths of $100 \mathrm{~m}$ and the surface, respectively. What knowledge we have about the ocean depths was obtained mainly through in-situ measurements conducted by a myriad of research vessels performing tens of thousands of casts of Nansen bottles, Niskin bottles, and CTD's. 
Though bottle and CTD casts are important and still continue today, they are time and labor intensive. Not only are they costly, but the slow sampling rate makes them susceptible to aliasing of many oceanic processes. As new methods have been sought out, the use of acoustics to probe the depths of the ocean has become an attractive, alternate technology in oceanic research. Acoustics has allowed man to determine accurately the topography of the ocean floor, to track marine life, to profile the ocean currents, and to perform many other useful tasks. One of the more recent research efforts in acoustics has been that of ocean acoustic tomography. The idea behind tomography is to take many slices or sections of an object at different angles to produce a three dimensional picture of the subject. The theory was mathematically proven by Radon in 1917 [cited in Munk and Wunsch, 1979]. Tomography has been widely used in medicine (CAT scan stands for Computer Assisted Tomography scan), and in seismology.

The basic principle behind ocean acoustic tomography is that by measuring the long-term, temporal variation in acoustic pulse travel times between a source and a receiver, due to the sound speed field variation from effects such as fronts and eddies, one can image these features acoustically, and thus study them. This temporal variation had been noted in several early experiments [see Hamilton, 1977; Steinberg and Birdsall, 1966 (both cited in Munk and Wunsch, 1979)], but was often considered noise. Worcester [1977] conducted one of the first experiments in ocean acoustic tomography, when he measured reciprocal acoustic travel times between two ships 25 $\mathrm{km}$ apart, in order to investigate the feasibility of using the travel time differences to monitor mid-ocean currents. Munk and Wunsch [1979] proposed expanding the range between source and receiver to $1000 \mathrm{~km}$ to monitor mesoscale ocean features. Since then, acoustic tomography has been applied to both small scale and large scale oceanographic eddies, as well as to shallow water and deep water environments. 


\subsection{Thesis Objectives}

In this work, we will first calculate internal tide and internal wave spectra and amplitudes from temperature measurements taken at moorings at several depths in the Barents Sea during the August 1992 Barents Sea Polar Front (BSPF) Experiment. Using this oceanographic data, the perturbation in acoustic travel times caused by the internal tides and internal waves is predicted. This will be eventually compared to actual perturbations in travel time recorded during the experiment. These perturbations are expected to be significant enough to be measurable.

\subsection{Thesis Content}

The scope of this thesis is as follows: Chapter two first presents the theory behind acoustic tomography, internal tides and internal waves. Next, using adiabatic normal mode theory, a formulation for the travel time perturbation of an acoustic signal due to internal waves is presented. Chapter three describes the region of the experiment, the experiment itself, and the data collected. Chapter four details the oceanographic data analysis conducted and reviews the assumptions which have been made. Chapter five examines travel time perturbations due to internal tides and internal waves. Comparisons between a simple ray model and the modal formulation derived in Chapter two are made. Finally, Chapter six presents conclusions and gives suggestions for future work. 


\section{Chapter 2}

\section{Theory}

\subsection{Acoustic Tomography}

Acoustic tomography can be broken into a forward and an inverse problem. In the forward problem, the travel time of a pulse along a path is computed from the sound speed and current fields of the ocean. In the inverse problem, given a set of travel times, the sound speed and current structure of the ocean is to be estimated. In this section, we will look at the ray theory approach to the forward problem.

The formulation of the forward problem given here follows that of Howe et al. [1987]. Using geometric optics, the travel time $T_{i}$ along a ray path $\Gamma_{i}$ in an ocean having a sound speed field $C(\mathbf{x}, t)$ and a current field $\mathbf{u}(\mathbf{x}, t)$ is

$$
T_{i}=\int_{\Gamma_{i}} \frac{d s}{C(\mathrm{x}, t)+\mathrm{u}(\mathrm{x}, t) \cdot \tau}
$$

where $s$ is the arc length, and $\tau$ is a unit vector tangent to the ray. The sound speed field $C(\mathbf{x}, t)$ can be decomposed as

$$
C(\mathbf{x}, t)=C_{0}(\mathbf{x})+\delta C(\mathbf{x}, t)
$$

where $C_{0}(\mathbf{x})$ is a "known" background sound speed field and $\delta C(\mathbf{x}, t)$ is a sound speed perturbation such that $\delta C(\mathbf{x}, t) \ll C_{0}(\mathbf{x})$. From these equations it can be seen that 
a ray traveling with a current has a smaller travel time than a ray traveling against the current.

The perturbation travel times for a reciprocal transmission between transceivers can be found by substituting (2.2) into (2.1) and linearizing. This gives

$$
\delta T_{i}^{+}=T_{i}^{+}-T_{0 i}=-\int_{\Gamma_{i}} \frac{\delta C(\mathbf{x}, t)+\mathbf{u}(\mathbf{x}, t) \cdot \boldsymbol{\tau}}{C_{0}^{2}(\mathbf{x})} d s,
$$

and

$$
\delta T_{i}^{-}=T_{i}^{-}-T_{0 i}=-\int_{\Gamma_{i}} \frac{\delta C(\mathbf{x}, t)-\mathbf{u}(\mathbf{x}, t) \cdot \tau}{C_{0}^{2}(\mathbf{x})} d s
$$

where the + superscript refers to a ray propagating in the $+\mathbf{x}$ direction and the - superscript is a ray propagating in the $-\mathrm{x}$ direction. By taking the sum $s_{i}$, or the difference $d_{i}$ of $(2.3)$ and $(2.4)$, either $\mathbf{u}(\mathbf{x}, t) \cdot \tau$, or $\delta C(\mathbf{x}, t)$ may be eliminated, respectively giving

$$
s_{i}=\delta T_{i}^{+}+\delta T_{i}^{-}=-\int_{\Gamma_{i}} \frac{2 \delta C(\mathbf{x}, t)}{C_{0}^{2}(\mathbf{x})} d s
$$

and

$$
d_{i}=\delta T_{i}^{+}-\delta T_{i}^{-}=-\int_{\Gamma_{i}} \frac{2 \mathbf{u}(\mathbf{x}, t)}{C_{0}^{2}(\mathbf{x})} d s
$$

Howe et al. [1987] showed that internal waves and large scale velocity shears do not significantly perturb the reference ray path so that for a two-way transmission the ray paths are reciprocal to first order and for a one-way transmission the ray paths for the reference ray and the perturbed ray are identical. In the BSPF experiment, only the one-way transmission case is examined. By noting that $\delta C(\mathbf{x}, t) \gg \mathbf{u}(\mathbf{x}, t) \cdot \tau$, (2.3) can be reduced to

$$
\delta T_{i}^{+}=T_{i}^{+}-T_{0 i}=-\int_{\Gamma_{i}} \frac{\delta C(\mathrm{x}, t)}{C_{0}^{2}(\mathrm{x})} d s .
$$




\subsection{Internal Motions in the Ocean}

Internal motions in the ocean of interest in this thesis are internal tides and internal waves. This classification is a matter of the frequency of interest. Internal tides are forced at tidal frequencies $\omega_{\text {tide }}$, and are either propagating or evanescent depending on whether $\omega_{\text {tide }}$ is greater than or less than the inertial frequency $f$, respectively. The propagating internal wave band is between the inertial frequency $f$ and the highest buoyancy frequency $N_{\max }$. These internal motions are clearly seen in Fig. 2-1, which is an example of a temperature record that has been has been Fourier transformed into its frequency components (using methods to be discussed later in Chapter 4). Clearly shown in the figure are the semidiurnal internal tide along with its harmonics, and the internal wave spectrum at higher frequencies. $N_{\max }$ at this depth is 215 cycles per day.

\subsubsection{Internal Tides}

Internal tides are generated by the surface or barotropic tides advecting water over the ocean bottom. Any change in the bottom topography causes the isopycnals to be displaced. This disturbance excites the internal modes in the water. The continental shelf break is a principal generation site of internal tides, whose generation mechanics have been well studied [e.g. Baines, 1974; Rattray et al. 1969]. Internal tides can also be generated at other topographic changes such as guyots, troughs, sills, and sea mounts [e.g. Maxworthy, 1979].

Wunsch [1975] reviewed several internal tide records and noted they often showed a quasi-semidiurnal or quasi-diurnal periodicity, but that due to the record length, background noise, and frequency shifting of the tidal peak, it was often hard to resolve the tidal constituents. He also noted that the tidal signal was intermittent. Baines [1986] suggested that the intermittent nature of the internal tide might be due to

- the changes in stratification between the generation site and the observer, or due to frontal changes or upwelling. The generation of internal tides is a very non-linear effect. Many records show periodicities of 6 hours (twice semidiurnal) or 8 hours 


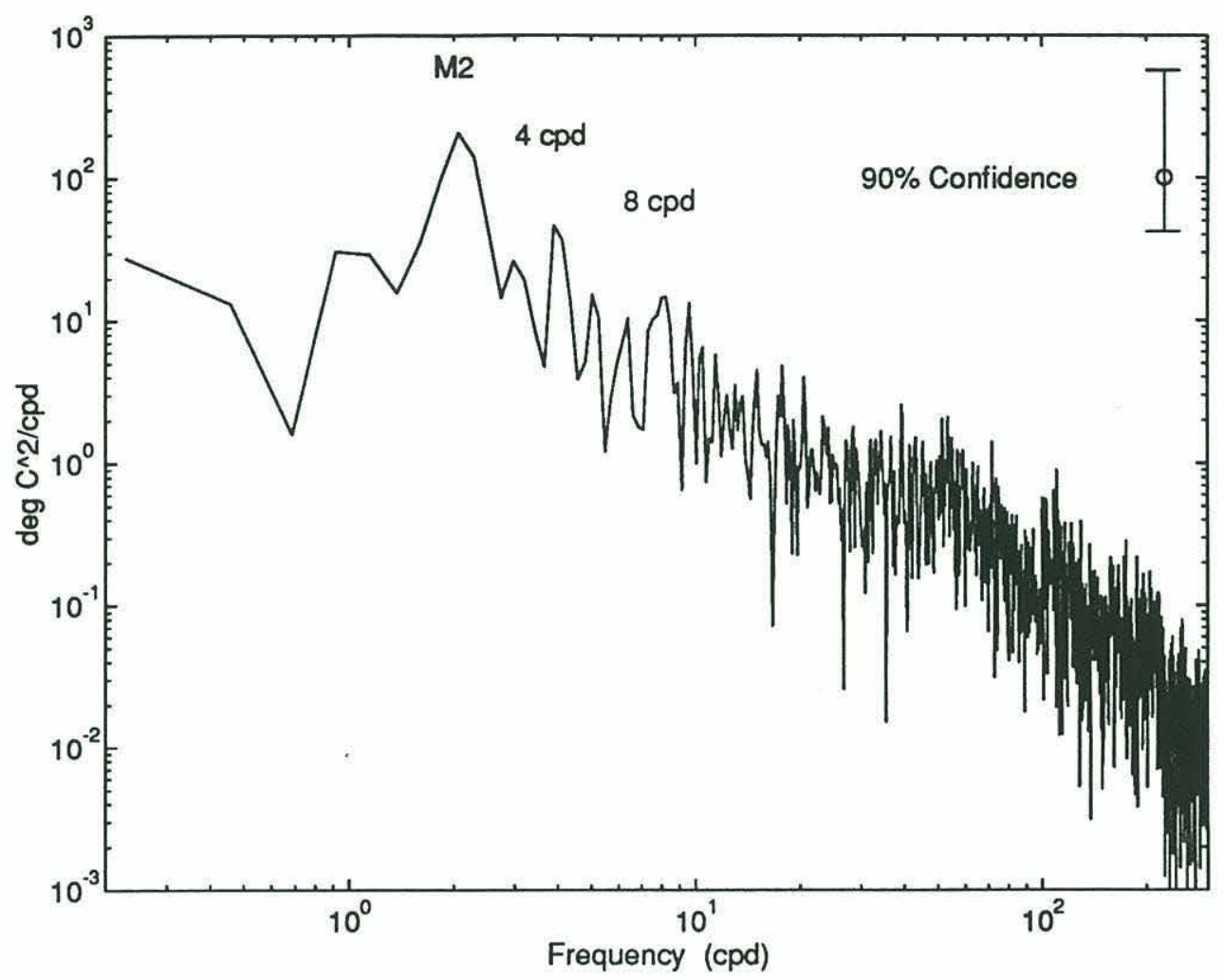

Figure 2-1: Power Spectrum Density of Brancker temperature logger \#3663 located on NE array at a depth of $32.1 \mathrm{~m}$. Evident are the M2 tide, its higher harmonics, and the internal wave band. $N_{\max }$ at this depth is $215 \mathrm{cpd}$. The estimate has 4 degrees of freedom.

(diurnal plus semidiurnal), or even 3 hours [Baines, 1986]. The 3 hour ( $8 \mathrm{cpd}), 6$ hour (4 cpd), and 12 hour ( $2 \mathrm{cpd}$ ) periods are evident in Fig. (2-1). Some of these higher harmonics of $M 2$ could be due to computing the spectrum of temperature, rather than density, however, as will be discussed.

In his review, Wunsch [1975] also noted that most of the internal tidal energy is contained in the first few lowest modes with mode one dominant. The direction of propagation is generally shoreward, but this need not be the case. For instance, Levine and Richman [1989] performed a study which showed that the internal tide off the coast of California propagated along the shelf. They suggested that the internal tides were either being refracted from fronts along the coast or from mesoscale structures, or that the resultant direction is due to many waves being radiated from several shelf 
breaks oriented in different directions. Another study by Holloway [1984] found that the first mode dominates and propagates onshore at an angle of about $30^{\circ}$ from normal to the bathymetry.

\subsubsection{Internal Waves}

Internal waves are found throughout the ocean. They exist in the ocean interior due to disturbances between fluids of differing densities. Although numerous internal wave generation mechanisms have been detailed, only the more important mechanisms will be discussed here. There exist several thorough reviews which the reader may wish to consult [Thorpe, 1975; Müller and Olbers, 1975; LeBlond and Mysak, 1978; Olbers, 1983]. The internal wave generation mechanisms involve interactions with the bottom, the surface, or the interior.

Bottom topography is an important mechanism in the formation of internal waves, particularly in coastal regions. This mechanism has been extensively studied in the region of the continental shelf break [Sawyer, 1983; Curtin and Mooers, 1975], over ridges in bays [Halpern, 1971; Chereskin, 1983] and over sills in straits [Gargett, 1976; Farmer and Smith, 1980]. In a horizontally stratified ocean, the flow of the baroclinic tide over a sharp boundary (such as a continental shelf or a ridge) causes a shear instability and creates a lee wave. Depending upon the specific conditions, the lee wave will either propagate shoreward as a lee wave train or evolve into an undular bore structure. These internal wave packets or solitons have generally been observed when the tide turns.

Surface interactions can also generate internal waves. Thorpe [1975] reviewed several surface interaction mechanisms, which we present here in order of assumed importance. First, the wind stress can produce a traveling stress field in the mixed layer, which results in a divergent current field in the mixed layer. The vertical Ekman velocities created by the wind stress can excite internal waves. Second, a traveling pressure field, created by atmospheric pressure, can resonantly couple with the internal waves to form waves that grow linearly. Third, a traveling buoyancy flux, produced by solar heating and precipitation, induces a vertical velocity at the lower 
boundary of the surface layer. The induced velocities excite internal waves. Fourth, a spectrum of surface gravity waves can be produced by the wind stress acting over the ocean surface. Two of these higher frequency surface gravity waves, $s 1$ and $s 2$ can beat against each other to produce a lower frequency internal wave with wave number $\mathbf{k}_{i w}$

$$
\mathbf{k}_{i w}=\mathbf{k}_{s 1}-\mathbf{k}_{s 2},
$$

and frequency $\omega_{i w}$

$$
\omega_{i w}=\omega_{s 1}-\omega_{s 2} .
$$

The interaction of the surface waves generates high frequency internal waves in the upper ocean, but this mechanism is insignificant for the deep ocean. Under conditions of a rough sea and a strong, shallow thermocline, however, it may be comparable to the wind stress mechanism (the first mechanism mentioned) [Olbers, 1983]. Finally, instability between the surface boundary layer and the deep ocean, such as turbulence in the mixed layer, may also generate internal waves.

As regards the role of the interior of the ocean in the generation of internal waves, very little is known. LeBlond and Mysak [1978] list as possible generation mechanisms processes such as penetrative convection, geostrophic adjustment, and internal tidal modulation of the thickness of the thermocline.

Garrett and Munk [1972] looked at several internal wave observations and created from them a model of the internal wave field frequency-wavenumber spectrum. The Garrett-Munk model assumes horizontal isotropy and vertical symmetry. It has the form

$$
S\left(k_{h}, \omega\right) \propto \mu^{-1} \omega^{-p+1}\left(\omega^{2}-f^{2}\right)^{-1 / 2}
$$

where $k_{h}$ is the horizontal wavenumber, $f$ is the local inertial frequency, and $\mu$ is the cut-off wavenumber which satisfies

$$
\mu \propto \omega^{r-1}\left(\omega^{2}-f^{2}\right)^{1 / 2} .
$$

Substituting in (2.11) into (2.10), assuming $\omega^{2} \gg f^{2}$, and then integrating (2.10) over 
$k_{h}$, gives for the frequency spectra

$$
S(\omega)=\omega^{-p} .
$$

The indices $p$ and $r$ are determined from observed spectra, which suggested values of $p$ between $5 / 3$ and 2, and $r$ between 2/3 and 3/2. Garrett and Munk proposed a value of 2 for both $r$ and $p$. The Garrett-Munk model has been modified and refined over the years as new experimental information has become available.

While the spectrum for the deep ocean is well modeled by Garrett-Munk, that for shallow waters is not. An internal wave experiment, conducted as part of the GARP Atlantic Tropical experiment or GATE in 1974, gave a spectrum which deviated strongly from the Garrett-Munk model [Käse and Siedler, 1980]. The frequency spectrum showed three dominant energy bands centered about the near-inertial frequency, the M2 tidal frequency, and 3 cycles per hour (cph). The high frequency wave field was intermittent and anisotropic. The non-stationarity of the spectra is a result of the variability of the atmospheric generation mechanisms [Käse and Clarke, 1978]. Käse and Clarke also argue that the the higher frequency waves are trapped near the surface where a maximum $N$ overlies a thick weakly stratified lower layer. These waves form a low order standing mode. Baines [1986] also noted a study in which for depths less $800 \mathrm{~m}, 25-45 \%$ of the energy was in mode 1 which propagated shoreward.

\section{Dynamical Equations for Internal Waves}

Using the basic equations of momentum and continuity, an exact modal solution for internal waves can be derived. The equation of momentum for an isentropic fluid in the absence of viscous effects is given by [e.g. Gill, 1982]

$$
\frac{D \mathbf{u}}{D t}+2 \Omega \times \boldsymbol{u}=-\rho^{-1} \nabla p-\mathbf{g} .
$$


and the continuity equation for an incompressible fluid is given by

$$
\boldsymbol{\nabla} \cdot \boldsymbol{u} \equiv \partial u / \partial x+\partial v / \partial y+\partial w / \partial z=0
$$

Following the derivation of Apel [1987], a small perturbation in $p$ and $\rho$ is applied to (2.13). The perturbation pressure and density are then given respectively by

$$
p=-g \rho z+p^{\prime},
$$

and by

$$
\rho=\rho_{0}+\rho^{\prime} .
$$

The momentum equation (2.13) is linearized by neglecting the products of perturbation quantities, and becomes

$$
\begin{gathered}
\frac{\partial u}{\partial t}-f v=-\frac{1}{\rho_{0}} \frac{\partial p^{\prime}}{\partial x}, \\
\frac{\partial v}{\partial t}-f u=-\frac{1}{\rho_{0}} \frac{\partial p^{\prime}}{\partial y}, \\
\frac{\partial w}{\partial t}=-\frac{1}{\rho_{0}} \frac{\partial p^{\prime}}{\partial z}-\frac{\rho^{\prime}}{\rho_{0}} g,
\end{gathered}
$$

where $f$ is the inertial or "local Coriolis" frequency. We now assume a separable solution for the vertical velocity $w(\mathbf{x})$ of the form

$$
w(\mathbf{x}, t)=\hat{w}(z) \exp [\imath(k x+l y-\omega t)],
$$

where $\hat{w}(z)$ is the vertical eigenfunction. The vertical velocity $w(\mathbf{x})$ satisfies the boundary conditions of no normal flow at the bottom

$$
w(\mathbf{x})=0 \text { at } z=h \text { (bottom), }
$$


and that a particle on the free surface remains on it

$$
w(\mathbf{x})=\partial \eta / \partial t \quad \text { at } z=\eta(\text { surface })
$$

which can be applied at $z=0$ since $\eta$ is small. This approximation introduces little error, as the vertical disturbance at the air-water boundary is much smaller than the maximum amplitude by an order of 1000. By manipulating equations (2.14-2.19), one can obtain

$$
\frac{\partial^{2} \hat{w}}{\partial z^{2}}+k_{h}^{2}\left[\frac{N^{2}(z)-\omega^{2}}{\omega^{2}-f^{2}}\right] \hat{w}=0,
$$

where $k_{h}$ is the horizontal wavenumber that satisfies

$$
k_{h}^{2}=k^{2}+l^{2},
$$

and where $N$ is the buoyancy or Brunt-Väisälä frequency which satisfies

$$
N^{2}=-\frac{g}{\rho_{0}} \frac{d \rho_{0}}{d z} .
$$

For $\omega<f$ or $\omega>N$, the vertical wave number is imaginary and the solutions to $(2.20)$ are evanescent.

By using the relationship

$$
\hat{w}=\frac{\partial \zeta}{\partial t},
$$

where $\zeta$ is the particle displacement, and substituting in (2.23) gives

$$
\frac{\partial^{2} \zeta}{\partial z^{2}}+k_{h}^{2}\left[\frac{N^{2}(z)-\omega^{2}}{\omega^{2}-f^{2}}\right] \zeta=0,
$$

with the following boundary conditions:

$$
\zeta(z)=0 \text { at } z=0 \text { (surface); }
$$

and

$$
\zeta(z)=0 \text { at } z=h \text { (bottom). }
$$


This equation can be formulated as an eigenvalue equation and is easily solved using numerical methods such as finite element methods. The exact modal solution is

$$
\zeta=\sum_{l=1}^{\infty} A_{l} \psi_{l}(z) \exp \left[\imath\left(k_{l} x+l_{l} y-\omega t\right)\right]
$$

where $l=1,2, \ldots$ is the mode number, $A_{l}$ is the amplitude, and $\psi_{l}(z)$ is the vertical internal wave mode eigenfunction.

\subsection{Adiabatic Acoustic Normal Modes}

We now return to acoustics. In a range independent environment, the acoustic pressure $p(z, \mathbf{x})$ for a point source at $r=0, z=z_{1}$ is described by the Helmholtz equation

$$
\nabla^{2} p+K^{2}(z) p=-\frac{2}{r} \delta\left(z-z_{1}\right) \delta(r)
$$

Brekhovskikh and Lysanov [1991] give as the solution

$$
p(r, z)=\pi \imath \sum_{m} \phi_{m}(z) \phi_{m}\left(z_{1}\right) H_{0}^{(1)}\left(K_{m} r\right) .
$$

The vertical mode function $\dot{\phi}_{m}$ satisfies

$$
\frac{d^{2} \phi_{m}}{d z^{2}}+\left[K^{2}(z)-K_{m}^{2}\right] \phi_{m}=0
$$

where $K(z)$ is the total wavenumber and $K_{m}^{2}$ is the $m^{\text {th }}$ modal eigenvalue. Equation (2.32) is a good approximation for many parts of the ocean, but as it is range independent, it is inadequate where the depth of the ocean changes significantly, where the sound waves cross ocean fronts, or where there is a significant change in the sound speed profile along the propagation path. In these cases the Helmholtz equation, equation (2.31), must be modified to take into account the variation of ocean parameters with range and becomes

$$
\nabla^{2} p+K^{2}(z, \mathbf{r}) p=0
$$


Shang [1989] gives as the solution to (2.34)

$$
p(r, z)=\sum_{n=1}^{\infty} \frac{F_{n}(r) \phi_{n}(z, r)}{\sqrt{r}}
$$

where $F_{n}(r)$ is the radial component and the vertical mode function $\phi_{n}(z, r)$ satisfies

$$
\frac{\partial}{\partial z}\left(\frac{1}{\rho_{0}(z)} \frac{\partial \phi_{n}}{\partial z}\right)+\left(\frac{K^{2}(z, r)}{\rho_{0}(z)}-\frac{K_{n}^{2}(r)}{\rho_{0}(z)}\right) \phi_{n}=0
$$

The mode functions $\phi_{n}$ are mutually orthogonal and satisfy

$$
\int_{0}^{h} \frac{1}{\rho_{0}} \phi_{m} \phi_{n}=\delta_{m n}
$$

Substituting (2.35) into (2.34), multiplying by $\phi_{m}$, and then integrating over $z$ from 0 to $h$ results in the following coupled equation

$$
\frac{d^{2} F_{m}}{d r^{2}}+\left(K_{m}^{2}(r)+\frac{1}{4 r^{2}}\right) F_{m}=-\sum_{n}\left[A_{m n}(r) F_{n}+2 B_{m n}(r)\right] \frac{d F_{n}}{d r}
$$

where $A_{m n}$ and $B_{m n}$ are coupling coefficients defined as

$$
\begin{aligned}
A_{m n} & =\int \rho_{0}^{-1} \phi_{m}(r, z) \frac{\partial^{2} \phi_{n}}{\partial r^{2}}(z, r) d z, \\
B_{m n} & =\int \rho_{0}^{-1} \phi_{m}(r, z) \frac{\partial \phi_{n}}{\partial r}(z, r) d z .
\end{aligned}
$$

To obtain the "adiabatic approximation", we neglect the $1 / 4 r^{2}$ term in (2.38) since we are interested in solutions with $K_{m} r \gg 1$, and we assume that the mode coupling effects are small. Physically, this means that the ocean properties (depth, sound speed) "vary slowly" as a function of horizontal range. The phrase "vary slowly" means that the fractional change of the ocean properties over a distance of less than a wavelength is small [Pierce, 1965]. This type of medium has been termed "almost stratified". The adiabatic approximation solution to (2.34) is

$$
p(r, z)=\sqrt{2 \pi} \exp (i \pi / 4) \sum_{m} \frac{\phi_{m}\left(z_{1} \mid 0\right) \phi_{m}(z \mid r)}{\sqrt{K_{m}(r) r}} \exp \left(\imath \int_{0}^{R} K_{m}\left(r^{\prime}\right) d r^{\prime}\right) .
$$


In the Barents Sea, the background sound speed profile and the depth vary reasonably slowly along the sound propagation path on either side of the front. Taking advantage of this we can crudely treat the path on either side of the front as a "nearly range independent" segment, and use the adiabatic approximation. We expect strong coupling of the modes, though, in going through the frontal region.

\subsection{Sound speed perturbations due to Internal Waves}

Equation (2.3) shows that the two mechanisms affecting the perturbation of the acoustic signal are the horizontal current $\mathbf{u}(\mathbf{x}, t) \cdot \tau$ and the sound speed perturbation $\delta C(\mathbf{x}, t)$ which is related to the particle displacement $\zeta$ using the following relationship

$$
\delta C=\frac{\partial C_{p}}{\partial z} \zeta
$$

where $C_{p}^{\prime}=\partial C_{p} / \partial z$ is the potential sound speed gradient and $\zeta$ is the solution to equation (2.27). Assuming that both mechanisms are due to internal waves, Essen et $a l$. [1983] showed that $\delta C$ has a much greater affect on the acoustic signal than does $\mathbf{u}(\mathbf{x}, t) \cdot \boldsymbol{\tau}$.

Following a recent derivation by Jin [1992], for a band of frequency $\omega$, equation $(2.30)$ can be generalized to

$$
\zeta=\sum_{l=1}^{\infty} \int_{f}^{N} \int_{0}^{2 \pi} a_{l}(\omega, \theta) \psi_{l}(\omega, z) \exp \left[\imath\left(k_{l}(\omega) x+l_{l}(\omega) y-\omega t\right)\right] d \omega d \theta
$$

where $a_{l}(\omega, \theta)$ is the internal wave amplitude spectrum for mode $l, \psi_{l}(\omega, z)$ is the internal wave mode function, $\theta$ is the azimuthal angle, and $k_{l}(\omega)$ and $l_{l}(\omega)$ are the internal wave eigenvalues that satisfy equation (2.24). The quantities $\psi_{l}(\omega, z), a_{l}(\omega, \theta)$, $k_{l}(\omega)$, and $l_{l}(\omega)$ are assumed to be range independent.

We next follow Rajan et al. [1987] and perturb the background acoustic wavenumber $K^{0}(r, z)$ by perturbing the sound speed profile. A new wavenumber $K(r, z)$ can 
then be written as

$$
K(r, z)=\omega /\left[C_{0}(r, z)+\delta C(r, z)\right] .
$$

Using standard perturbation theory, a perturbation eigenvalue $\delta K_{m}(r)$ for $(2.41)$ is found to be

$$
\delta K_{m}(r)=\frac{1}{2 K_{m}^{(0)}(r)} \int_{0}^{\infty} \phi_{m}^{(0)}(r, z)\left|\delta K(r, z)^{2}\right| \phi_{m}^{(0)}(r, z) d z
$$

The middle factor in the integral in (2.45) can be rewritten as

$$
\begin{aligned}
\delta K(r, z)^{2} & =K^{2}(r, z)-K^{(0) 2}(r, z) \\
& =\frac{\omega^{2}}{C_{0}^{2}(r, z)}\left[\left(1+\frac{\delta C(r, z)}{C_{0}(r, z)}\right)^{-2}-1\right] \\
& \cong-K^{(0) 2}(r, z)\left(2 \delta C / C_{0}\right)
\end{aligned}
$$

Substituting (2.46) into (2.45) results in

$$
\delta K_{m}(r)=\frac{1}{K_{m}^{(0)}(r)} \int_{0}^{\infty} \rho^{-1}(z)\left|\phi_{m}^{(0)}(r, z)\right|^{2}\left(K^{(0)}(r, z)\right)^{2} \frac{\delta C(r, z)}{C_{0}(r, z)} d z
$$

Using the WKB approximation, the adiabatic modal phase is

$$
\varphi(r)=\int_{0}^{R} K_{m}(r) d r
$$

and the background modal phase is

$$
\varphi^{0}(r)=\int_{0}^{R} K_{m}^{0}(r) d r=K_{m}^{0} r .
$$

The modal phase perturbation is thus

$$
\delta \varphi_{m}=\int_{0}^{R} \delta K_{m}(r) d r
$$


If the received signal is processed by a narrow band filter with center frequency $\omega_{0}$, then the group travel time of each modal pulse is given by the stationary time as

$$
t_{m}=\frac{d\left[\varphi_{m}\right]}{d \omega}
$$

so that the group travel time perturbation is

$$
\delta t_{m}=\frac{d\left[\delta \varphi_{m}\right]_{\omega_{0}}}{d \omega}
$$

Inserting (2.47) into (2.50), and (2.50) into (2.52) and using the results of equation (18) of Lynch et al. [1991] results in

$$
\delta t_{m}=\int_{0}^{R} \int_{0}^{\infty} G_{m}(r, z) \delta C(r, z) d r d z
$$

where $G_{m}(r, z)$, the group velocity dispersion, is

$$
G_{m}(r, z)=\rho_{0}^{-1} \frac{1}{K_{m}^{(0)} C_{0}^{3}}\left[\left(2 \omega_{0}-\frac{C_{m}^{(0)}(r)}{U_{m}^{(0)}(r)} \omega_{0}\right)\left|\phi_{m}^{(0)}(r, z)\right|^{2}+\omega_{0}^{2} \frac{d}{d \omega}\left|\phi_{m}^{(0)}(r, z)\right|^{2}\right],
$$

and $C_{m}^{(0)}(r)$ and $U_{m}^{(0)}(r)$ are the modal phase and group velocities, respectively, for the unperturbed case. By assuming range independence, and also that

$$
\frac{C_{m}^{(0)}(r)}{U_{m}^{(0)}(r)} \approx 1
$$

(2.54) becomes

$$
G_{m}(z)=\frac{1}{C_{0}^{2}}\left[\left|\phi_{m}^{(0)}(z)\right|^{2}+\omega_{0} \frac{d}{d \omega}\left|\phi_{m}^{(0)}(z)\right|^{2}\right] .
$$

where $\rho(z)=1$ and $C_{m}^{(0)} \approx \bar{C}_{0}$. The bar denotes the average sound speed.

The covariance of $\delta t_{m}$ is formed by

$$
\operatorname{cov}_{m}(R, \tau)=\left\langle\delta t_{m}(R, t) \delta t_{m}^{*}(R, t+\tau)\right\rangle,
$$


which using (2.53) becomes

$$
\begin{aligned}
\operatorname{cov}_{m}(R, \tau)= & \int_{0}^{R} \int_{0}^{R} \int_{0}^{\infty} \int_{0}^{\infty} G_{m}(r, z) G_{m}\left(r^{\prime}, z^{\prime}\right) \times \\
& \left\langle\delta C(r, z, t) \delta C\left(r^{\prime}, z^{\prime}, t+\tau\right)\right\rangle d r d r^{\prime} d z d z^{\prime},
\end{aligned}
$$

where from (2.42) and (2.43)

$$
\begin{aligned}
\delta C(x, y, z, t)= & C_{p}^{\prime}(z) \sum_{l=1}^{\infty} \int_{f}^{N} \int_{0}^{2 \pi} a_{l}(\omega, \theta) \psi_{l}(\omega, z) \times \\
& \exp \left[\imath\left(k_{h l}(\omega) x \cos (\theta)+k_{h l}(\omega) y \sin (\theta)-\omega t\right] d \omega d \theta .\right.
\end{aligned}
$$

Assuming that the amplitudes $a_{l}(\omega, \theta)$ are independent, random variables with zero means implies that

$$
\left\langle a_{l}(\omega, \theta)\right\rangle=0
$$

and

$$
\left\langle a_{l}(\omega, \theta) a_{l^{\prime}}^{*}(\omega, \theta)\right\rangle=\left|a_{l}(\omega, \theta)\right|^{2} \delta_{\omega \omega^{\prime}} \delta_{\theta \theta^{\prime}} \delta_{l l^{\prime}},
$$

where $\delta_{\omega \omega^{\prime}}, \delta_{\theta \theta^{\prime}}$, and $\delta_{l l^{\prime}}$ are three delta functions. Additionally, if it is assumed that the $\mathrm{x}$ axis is in the direction from the source to receiver, we obtain

$$
\begin{aligned}
& \left\langle\delta C(r, z, t) \delta C\left(r^{\prime}, z^{\prime}, t+\tau\right)\right\rangle= \\
& \quad \sum_{l=1}^{\infty} \int_{f}^{N} \int_{0}^{2 \pi}\left|a_{l}(\omega, \theta)\right|^{2} \psi_{l}(\omega, z) \psi_{l}^{*}\left(\omega^{\prime}, z^{\prime}\right) \times \\
& \quad C_{p}^{\prime}(z) C_{p}^{\prime}\left(z^{\prime}\right) \exp \left[\imath\left(k_{h l}(\omega) \cos \theta\left(r-r^{\prime}\right)-\omega \tau\right)\right] d \omega d \theta .
\end{aligned}
$$

Substituting (2.60) into (2.58) results in

$$
\operatorname{cov}_{m}(R, \tau)=\sum_{l=1}^{\infty} \int_{f}^{N} \int_{0}^{2 \pi}\left|a_{l}(\omega, \theta)\right|^{2} p_{l}(\omega, \theta) e^{-\imath \omega \tau} d \omega d \tau
$$


where

$$
\begin{aligned}
p_{l}(\omega, \theta)= & \int_{0}^{\infty} \int_{0}^{\infty} \psi_{l}(\omega, z) \psi_{l}^{*}\left(\omega^{\prime}, z^{\prime}\right) C_{p}^{\prime}(z) C_{p}^{\prime}\left(z^{\prime}\right) \times \\
& {\left[\int_{0}^{R} \int_{0}^{R} G_{m}(r, z) G_{m}\left(r^{\prime}, z^{\prime}\right) \exp \left[\imath k_{h l}(\omega) \cos \theta\left(r-r^{\prime}\right)\right] d r d r^{\prime}\right] d z d z^{\prime} . }
\end{aligned}
$$

If $G_{m}(r, z)$ is assumed to be range independent, then it can be taken outside the integral and (2.62) reduces to

$$
p_{l}(\omega, \theta)=\int_{0}^{R} \int_{0}^{R} \exp \left[2 k_{h l}(\omega) \cos \theta\left(r-r^{\prime}\right)\right] d r d r^{\prime}\left|\int_{0}^{\infty} \psi_{l}(\omega, z) C_{p}^{\prime}(z) G_{m}(z) d z\right|^{2}
$$

The first integral on the right hand side of (2.63) reduces to

$$
\frac{2\left[1-\cos \left(k_{h l} \cos \theta R\right)\right]}{\left(k_{h l} \cos \theta\right)^{2}},
$$

and can be further reduced to

$$
R^{2} \operatorname{sinc}^{2}\left(\frac{k_{h l} R}{2} \cos \theta\right)
$$

where $\operatorname{sinc}(x) \equiv \sin x / x$. Thus (2.63) becomes

$$
p_{l}(\omega, \theta)=R^{2} \operatorname{sinc}^{2}\left(\frac{k_{h l} R}{2} \cos \theta\right)\left|\int_{0}^{\infty} \psi_{l}(\omega, z) C_{p}^{\prime}(z) G_{m}(z) d z\right|^{2}
$$

The covariance spectrum can be represented by

$$
F_{m}(R, \Omega)=\int_{-\infty}^{\infty} \operatorname{cov}_{m}(R, \tau) e^{-\imath \Omega \tau} d \tau
$$

Inserting (2.61) into (2.65) yields

$$
F_{m}(R, \Omega)=\sum_{l=1}^{\infty} \int_{f}^{N} \int_{0}^{2 \pi}\left|a_{l}(\omega, \theta)\right|^{2} p_{l}(\omega, \theta)\left(\int_{-\infty}^{\infty} e^{z(\Omega-\omega) \tau} d \tau\right) d \omega d \theta
$$


which after integrating over $\tau$ gives

$$
F_{m}(R, \Omega)=\sum_{l=1}^{\infty} \int_{f}^{N} \int_{0}^{2 \pi}\left|a_{l}(\omega, \theta)\right|^{2} p_{l}(\omega, \theta) \delta(\Omega-\omega) d \omega d \theta
$$

which can be reduced to

$$
F_{m}(R, \Omega)=\sum_{l=1}^{\infty} \int_{0}^{2 \pi}\left|a_{l}(\Omega, \theta)\right|^{2} p_{l}(\Omega, \theta) d \theta
$$

The previous results can be simplified by assuming that

$$
\left|a_{l}(\omega, \theta)\right|^{2}=A(\omega) B(\theta) D_{l},
$$

and further simplified by assuming $B(\theta)=\delta\left(\theta-\theta_{0}\right)$ and that the packet width of internal waves is $R_{d}$. For the case of $\theta_{0}=\pi / 2,(2.61)$ becomes

$$
\operatorname{cov}_{m}(R)_{\tau=0}=R_{d}^{2} \int_{f}^{N}\left[\sum_{l} A_{l}(\Omega) D_{l}\left|\int_{0}^{H} \psi_{l}(\Omega, z) C_{p}^{\prime}(z) G_{m}(z) d z\right|^{2}\right] d \Omega
$$

In this thesis we will evaluate equations (2.53) and (2.70). The assumptions we make in evaluating those equations are detailed in Section 4.3. 


\section{Chapter 3}

\section{Barents Sea Polar Front Experiment}

The Barents Sea Polar Front (BSPF) experiment took place from August 6 to August 26, 1992 in the coastal waters off of Svalbard, about 42 nautical miles due east of Bjørnøya or Bear Island at the northern edge of the Bjørnøya Trough. The BSPF experiment involved three CTD and ADCP surveys, and the deployment of four moorings for about ten days. Water depth in the experimental area varied from $110 \mathrm{~m}$ to $380 \mathrm{~m}$.

\subsection{Barents Sea}

The Barents Sea is a shallow, peripheral basin bounded by Svalbard on the north, Norway and Russia on the south, Novaya Zemlya on the east, and the Norwegian Sea on the west. The majority of the sea is contained between $70^{\circ}$ and $80^{\circ}$ north latitude and $16^{\circ}$ and $60^{\circ}$ east longitude (Fig. 3-1). The boundary between the Norwegian Sea and the Barents Sea is the transect from Spitsbergen to Bear Island to the coast of Norway. Table 3.1 gives the characteristics of the waters discussed below. For a detailed description of the water masses and types see Hopkins [1991] or Johannessen [1986]. The Barents Sea Polar Front (BSPF) is formed at the boundary of two currents, the North Cape Current to the south, and the Bear Island Current to the 


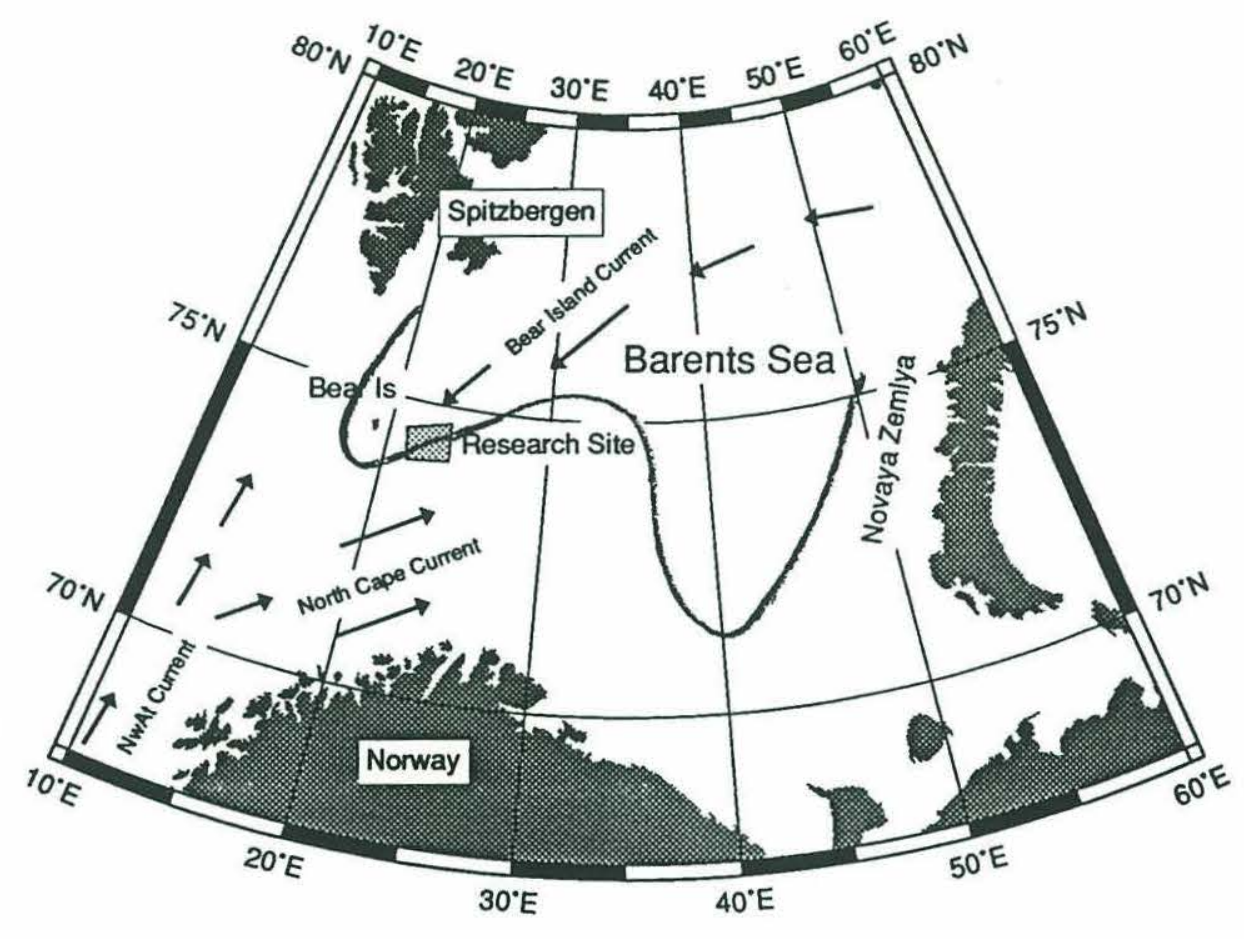

Figure 3-1: Chart of the Barents Sea with major currents. The thick, solid line is the Barents Sea Polar Front. 
Table 3.1: Water Mass Definitions

$\begin{array}{lrrrr}\text { Water Mass } & \underline{\text { Symbol }} & \underline{T\left({ }^{\circ} \mathrm{C}\right)} & \underline{S(\mathrm{ppt})} & \underline{\text { Comments }} \\ \text { North Atlantic Water } & \text { NAtW } & >8 & >35.3 & \begin{array}{r}\text { Surface water of } \\ \text { N. Atlantic Current }\end{array} \\ \text { Norwegian Atlantic Water } & \text { NwAtW } & >2 & >35.0 & \begin{array}{r}\text { Water of Norwegian } \\ \text { Atlantic Current }\end{array} \\ \text { Polar Water } & \text { PW } & \text { Freezing } & 33.0-34.4 & \\ \text { Barents Polar Water } & \text { BrPW } & -1-3 & <34.3 & \end{array}$

north. The North Cape Current, an extension of the Norwegian Atlantic Current (NwAtC), carries warm, saline Norwegian Atlantic Water (NwAtW) into the Barents Sea. The Bear Island Current, which flows along the northern part of the Bjørnøya or Bear Island Trough, carries cold, fresh Barents Polar Water (BrPW) out into the Norwegian Sea. The front is a stable interface because the warm, saline NwAtW has approximately the same density as the cold, fresh BrPW. The BSPF is some 1500 kilometers long and roughly follows the 200 meter isobath. It is also strongly influenced by the tidal cycle and can be displaced by a much as 10 kilometers during a tidal cycle [Johannessen 1986].

The tides in the Barents Sea are primarily semidiurnal $\left(M_{2}\right)$ with a weaker diurnal component $\left(K_{1}\right)$. Both Gjevik [1990] and Schwiderski [1986] have proposed tidal models for the Barents Sea. The predicted amplitudes for each model are given in table 3.2. The models differ in that Gjevik's model resolves amphidromes for both the $M_{2}$ and $K_{1}$ in the region of Bear Island, whereas Schwiderski's does not. Huthnance [1981] made a series of current meter measurements from Northern Norway to Bear Island. From his experiment, he estimates that both the $M_{2}$ and $K_{1}$ are mostly barotropic (about $86 \%$ ). The predominantly barotropic $S_{2}$ tide is smaller than the $M_{2}$, and has a fairly consistent relation with it. 
Table 3.2: Tidal Amplitudes at Bear Island

$\begin{array}{crrr} & \text { Obs. } & \begin{array}{r}\text { Gjev } \\ \text { Constituent }\end{array} & \begin{array}{r}\text { Amp. } \\ \text { Amp. }\end{array} \\ \begin{array}{c}\text { (cm) } \\ M_{2}\end{array} & 34.2 & 34.5 & \begin{array}{r}\text { Amp. } \\ (\mathrm{cm})\end{array} \\ K_{1} & 5.4 & 6.3 & 40 \\ & & & 5.5\end{array}$

Obs. - Observed values taken from Gjevik [1990] .

Gjev — Values taken from Gjevik [1990]

Schw — Values taken from Schwiderski [1990] .

\subsection{Experimental Objectives}

The purpose of the experiment was to study the structure and the dynamics of the Barents Sea Polar Front by using a variety of moored and shipboard oceanographic instruments. A key aspect of the BSPF experiment was the Barents Sea Tomography Transmission Test (BSTTT) whose major goals were: first, to determine the identifiability, resolvability, strength and variability of the multipath structure between the acoustic moorings; second, to examine the acoustic signature of various oceanographic processes (surface waves, internal tides, internal waves, eddies, etc.) between the acoustic moorings; and third, to use "tomographic inverse images" to study the region of the BSPF.

\subsection{Data Acquisition}

Four acoustic instruments were deployed during the experiment in a trapezoidal shape about 50 kilometers apart at the base, with the distance between the northeast and southeast moorings being 34 kilometers (Fig. 3-2). The Northwest (NW) and Southwest (SW) moorings contained $400 \mathrm{~Hz}$ tomography transceivers while the Northeast (NE) mooring contained a $224 \mathrm{~Hz}$ projector. These three moorings were deployed for a period of 9 days (NE) to 12 days (SW). The transceiver on the NW mooring acted as $400 \mathrm{~Hz}$ receiver only, while the transceiver on the SW mooring acted intermittently as a $400 \mathrm{~Hz}$ source and continuously as a $400 \mathrm{~Hz}$ receiver. The fourth or Southeast 


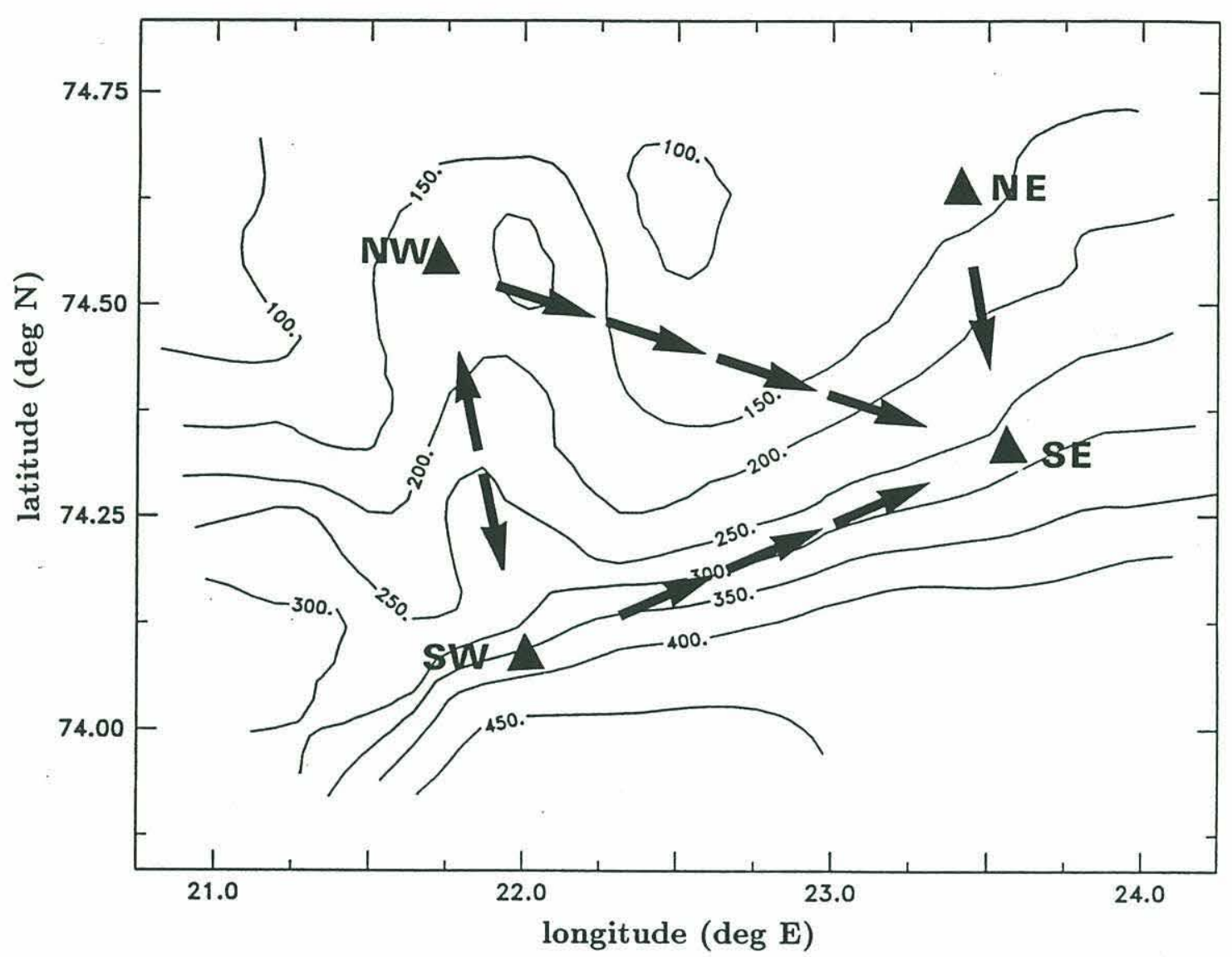

Figure 3-2: Chart of the BSPF experiment. Triangles are array positions. Arrows denote the acoustic paths during the experiment.

(SE) mooring had a vertical line array(VLA) of sixteen hydrophones which collected 70 hours of data. The data from the VLA was telemetered in real time back to the research ship using a wireless local area network. The acoustic paths between the moorings are shown in Fig. 3-2. Details of the vertical line array are given in von der Heydt et al. [1992]. All of the moorings except the SW mooring contained several current meters and Brancker temperature loggers to provide a vertical profile of current and temperature at each mooring. Details are given in Table 3.3.

Mooring motion of the source or receiver due to tides or other currents changes the source-receiver range, and introduces error into the measurement of the travel time. To correct for this mooring motion, all of the moorings except for the NE mooring used acoustic navigation systems. The determination of mooring motion is 


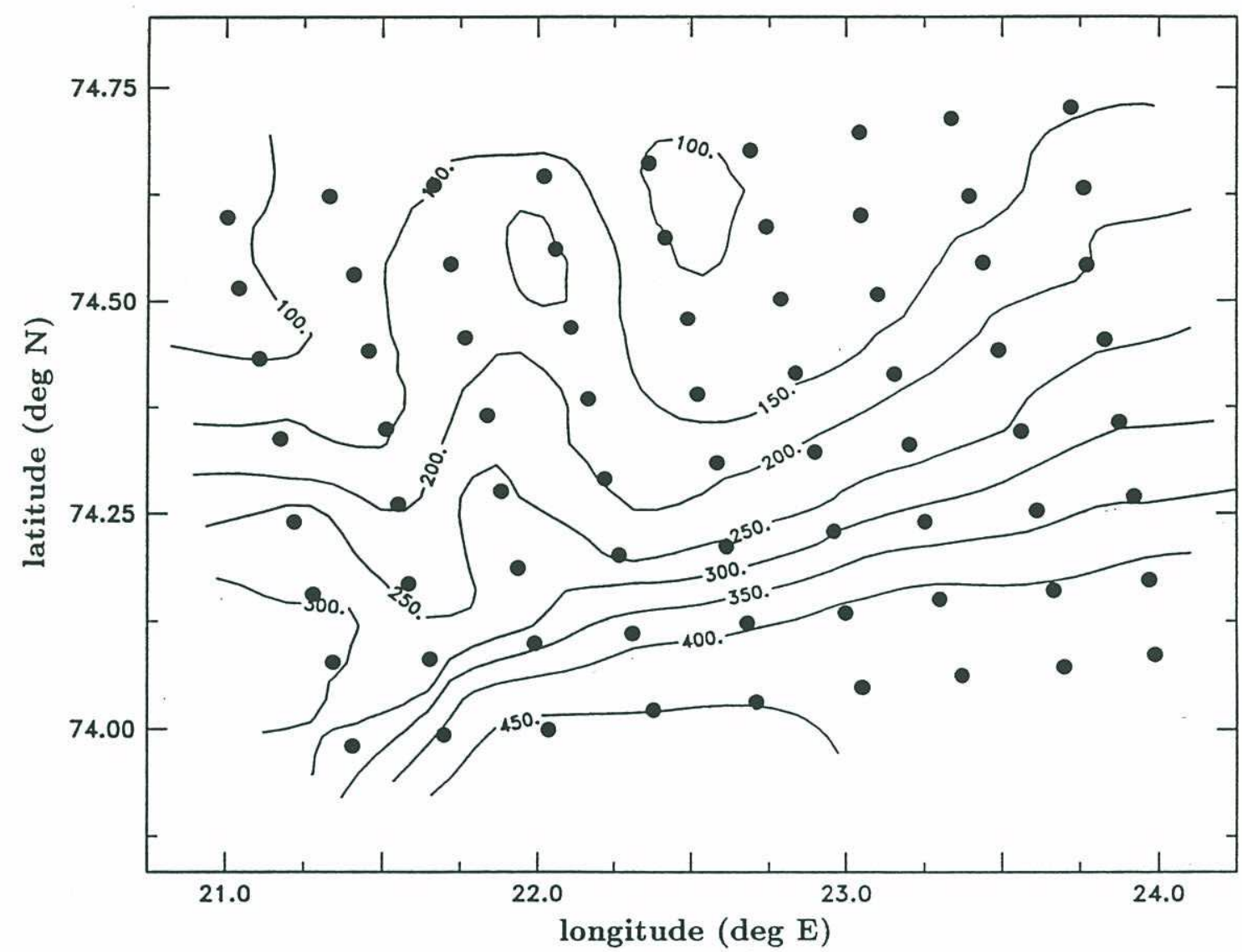

Figure 3-3: CTD grid employed during BSPF experiment. Circles are CTD stations. The grid was fully covered three times.

accurate to within $1 \mathrm{~m}$. Additionally, the SE mooring had two tilt meters. The ship also conducted three complete $70 \mathrm{~km}$ by $80 \mathrm{~km}$ CTD grids (Fig. 3-3) of the research area, two detailed grids across the Barents sea polar front, and a $24 \mathrm{hr}$ CTD anchor station at the SW mooring. Details of the CTD grids and the anchor station are given in Pawlowicz [1992]. Several SUS charges were also dropped to allow determination of bottom properties and reverberation characteristics. 
Table 3.3: Barents Sea Moorings

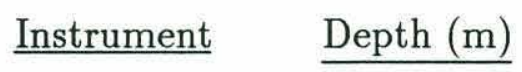

Northwest Mooring-170m

$\begin{array}{lr}\text { Neil Brown SACM } & 19.4 \\ \text { Brancker Tlog } & 29.5 \\ \text { Brancker Tlog } & 39.2 \\ \text { Aanderaa CM } & 49.3 \\ \text { 400 Hz transc. } & 66.7 \\ \text { Aanderaa CM } & 159.9\end{array}$

Northeast Mooring-142m

Neil Brown ACM

Brancker Tlog

32.1

Brancker Tlog

42.1

Neil Brown ACM

52.0

Aanderaa CM

82.1

$224 \mathrm{~Hz}$ proj.

Southwest Mooring-380m

Neil Brown SACM

19.9

Brancker Tlog

29.9

Brancker Tlog

39.9

Aanderaa CM

50.0

Aanderaa CM

80.0

Brancker Tlog

140.0

Brancker Tlog

200.5

$400 \mathrm{~Hz}$ transc.

358.1

Southeast Mooring-280m

Vertical Array $\quad 126-275.8$ 


\section{Chapter 4}

\section{Temperature Field Data Analysis}

The raw data from which the calculations in this chapter are made comes mainly from the CTD casts made at each mooring site, and the moored Brancker temperature loggers. Some temperature information is also from sensors in the current meters. Figure 4-1 is a typical Brancker temperature logger record. The temperature record is non-linear in that at higher temperatures the signal is "spikier" than at lower temperatures. This effect is most pronounced for those loggers located where the temperature gradient is most rapidly changing. A one meter downward displacement of water at the "knee" of the temperature curve (i.e. the mixed layer base) results in a much greater change of temperature than a one meter upward displacement. Figure 4-2(a) shows a sinusoidal 9.9m amplitude particle displacement which, acting on a typical temperature profile (Fig. 4-2(b)) results in a "distorted" temperature record (Fig. 4-2(c)).

The changes in temperature seen are due to both a vertical particle displacement of water caused by internal tides and waves, and a horizontal advection of water caused by the barotropic tide. Which mechanism is dominant can be determined by evaluating constraints imposed by the local temperature gradient, current velocities, and water depth. Figure 4-3 shows three CTD temperature profiles taken at the Southwest array. If we take profile \#418 as the baseline, and translate profile \#218 vertically by $+7 \mathrm{~m}$, then profile \#218 will lie directly over profile \#418. This corresponds to a temperature change solely due to internal tides and internal waves. 


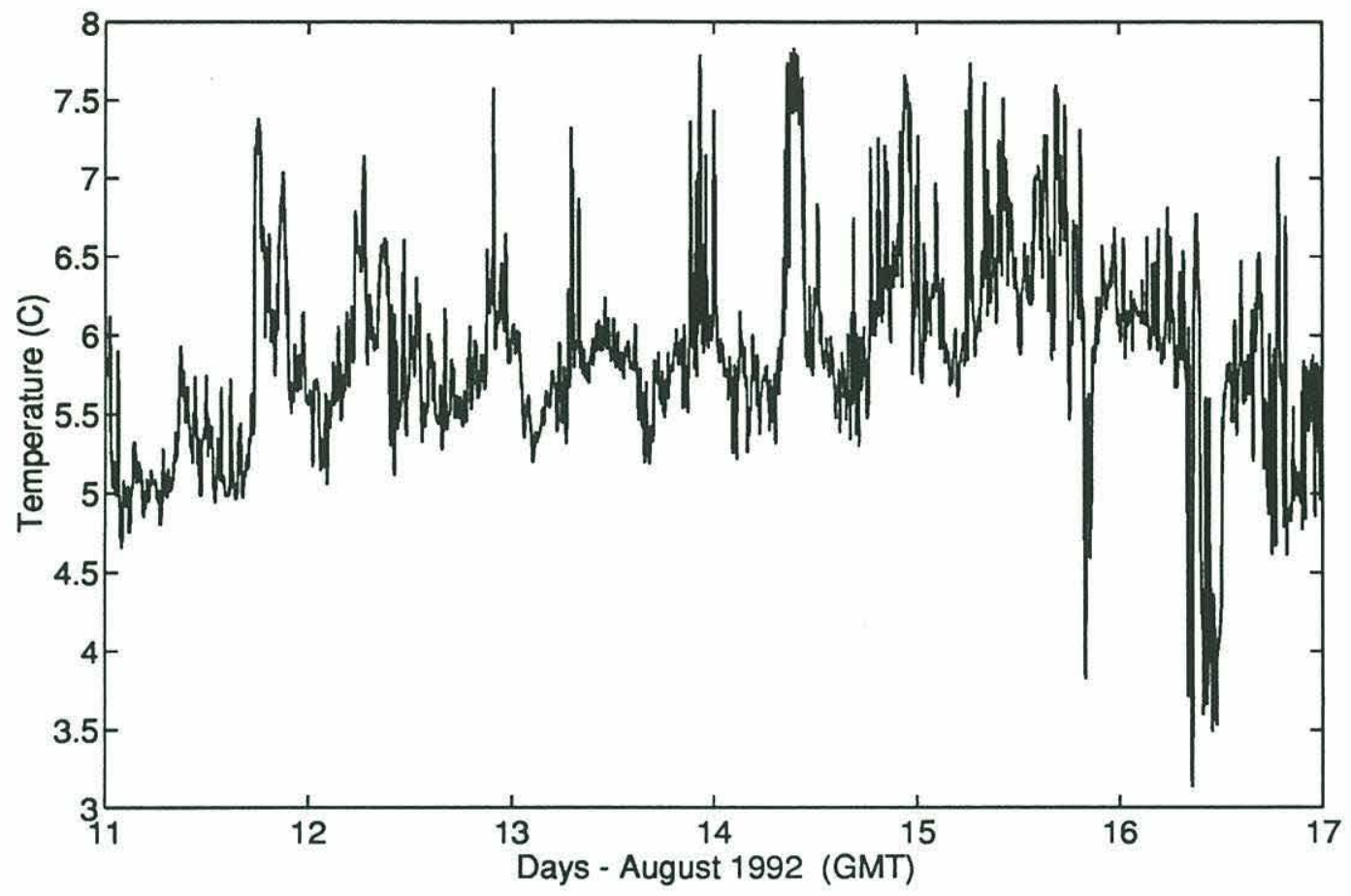

Figure 4-1: Temperature record from Brancker logger \# 3666 located on the SW mooring at a depth of $29.9 \mathrm{~m}$. Brancker temperature resolution is $0.1{ }^{\circ} \mathrm{C}$

Similarly, if profile \#118 is translated upwards in depth by $15 \mathrm{~m}$ and moved horizontally by $0.25{ }^{\circ} \mathrm{C}$ it will lie over profile \#418. This corresponds to a temperature change due to both a barotropic current and internal tides and internal waves. We will now consider the different effects separately, and thereby determine the percentage that each signal contributes to the whole.

\subsection{Horizontal Advection versus Internal Tides and Waves}

The amount of horizontal advection was determined at each of the three instrumented arrays by using the assumption that the temperature changes were solely due to horizontal advection and that any vertical motion due to internal tides and waves or topographic changes was negligible. In order to explain the method, the data from the NE mooring will be used. Figure 4-4 shows the temperature and velocity 

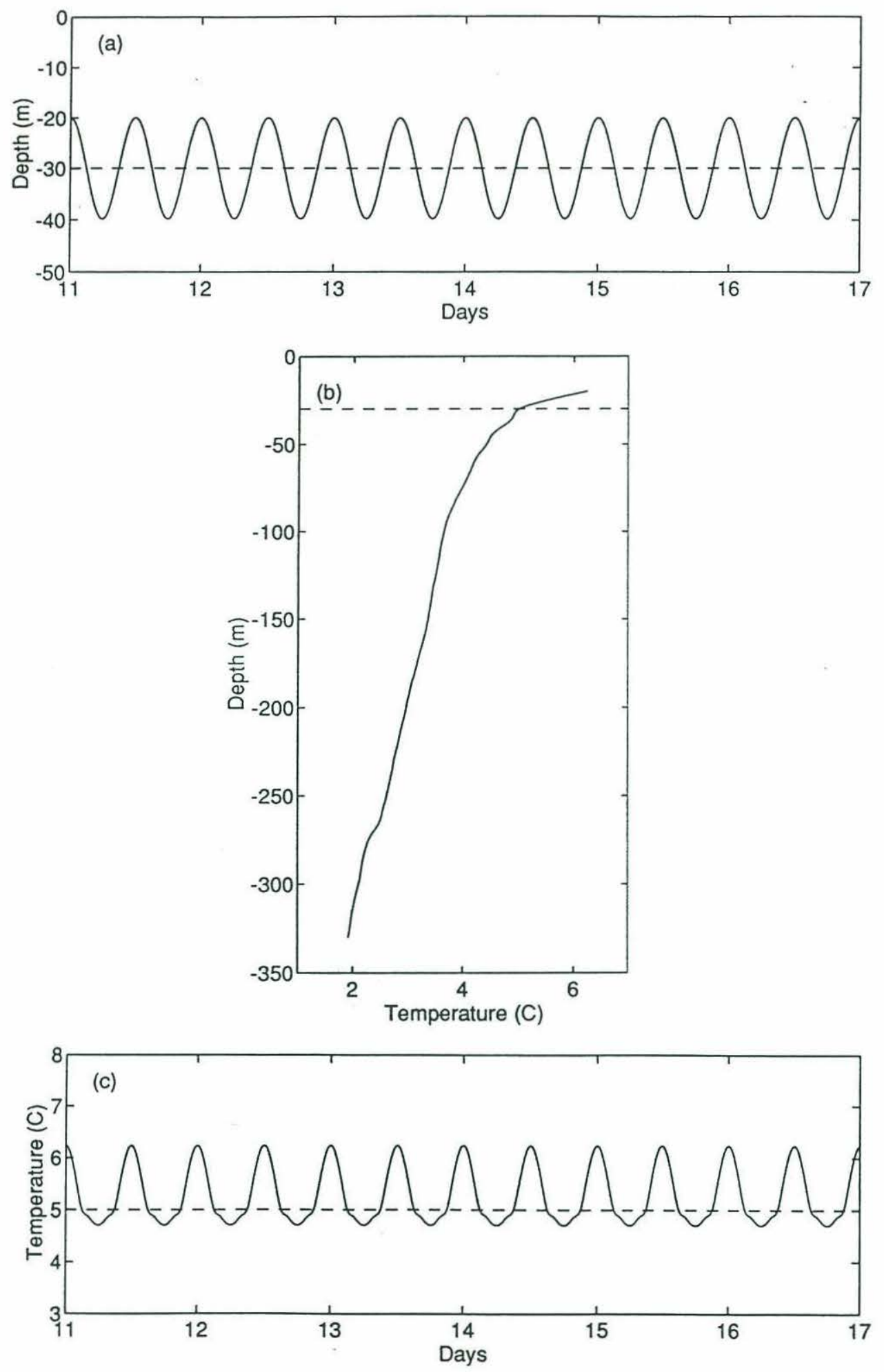

Figure 4-2: (a) is a $9.9 \mathrm{~m}$ sinusoidal particle displacement about the logger depth (dashed line). (b) is a smoothed temperature profile at the SW mooring. The dashed line is the logger depth. (c) is the "distorted" temperature record which results from the sinusoidal particle displacement in a varying temperature gradient. The dashed line is the axis of zero phase. 


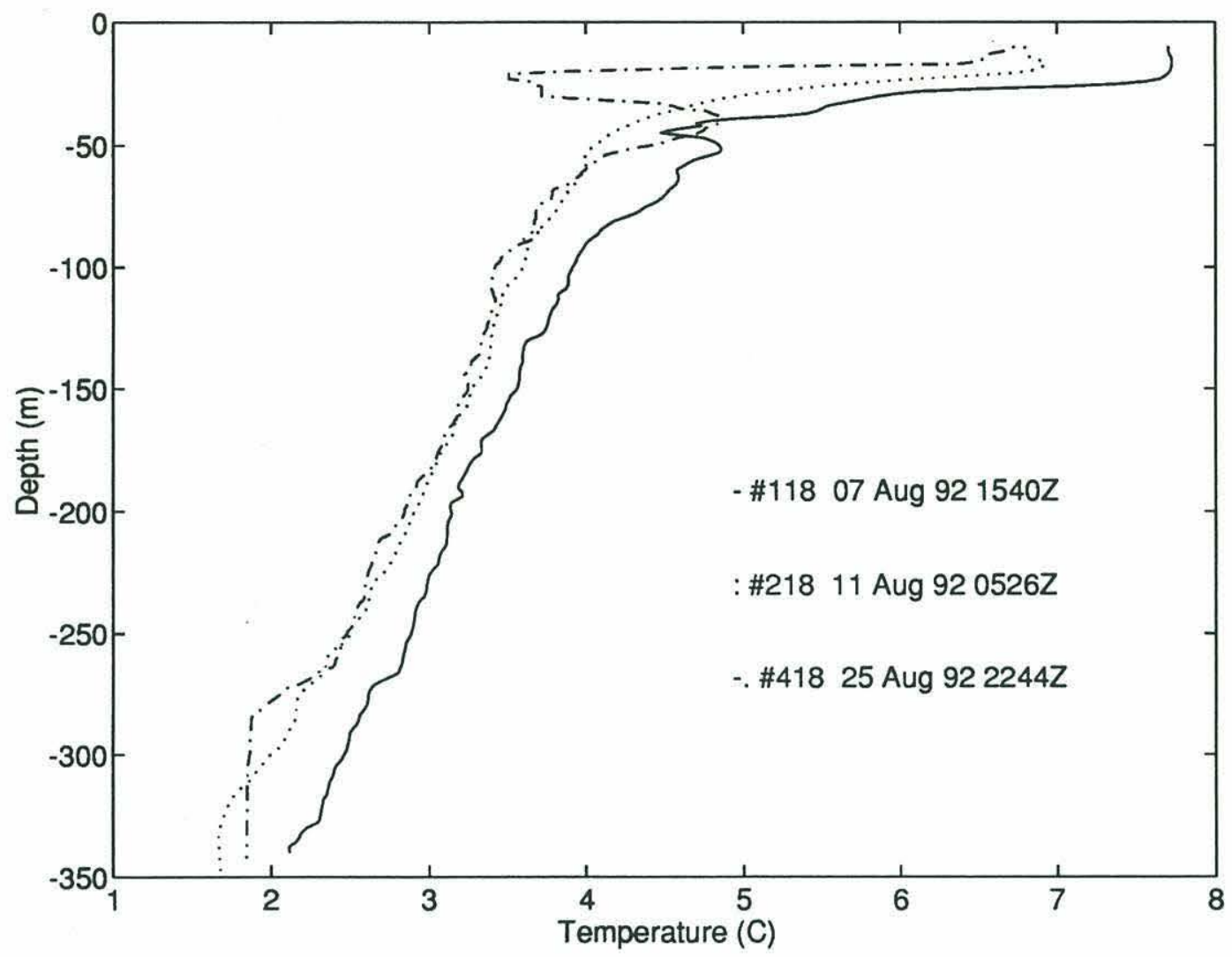

Figure 4-3: Three temperature profiles at the SW array taken over a span of 18 days. The temperature records show both a temperature change due to vertical displacement (caused by internal tides and waves) and due to horizontal advection (caused by the barotropic tide)

records from Aanderaa \#9259 at a depth of 82.1 meters. The temperature record has a minimum of $-0.9{ }^{\circ} \mathrm{C}$ and a maximum of $-0.36^{\circ} \mathrm{C}$ with a peak to peak change in temperature of $0.208^{\circ} \mathrm{C}$.

Figure 4-5 is a contour map of temperatures taken from the CTD profiles surrounding the NE mooring and shows that the warmer water at 82 meters is to the east and to the south, with the east-west gradient being much larger than the northsouth gradient. Several things are apparent in Fig. 4-4. The $u$ velocity lags the temperature record by 90 degrees, while the $v$ velocity is in phase with the temperature record. Given that the $u$ velocity is over twice the $v$ velocity and given that the east to west temperature gradient is much larger than the north south temperature gradient, the predominant effect will be from the $u$ velocity. Since the $u$ velocity lags 

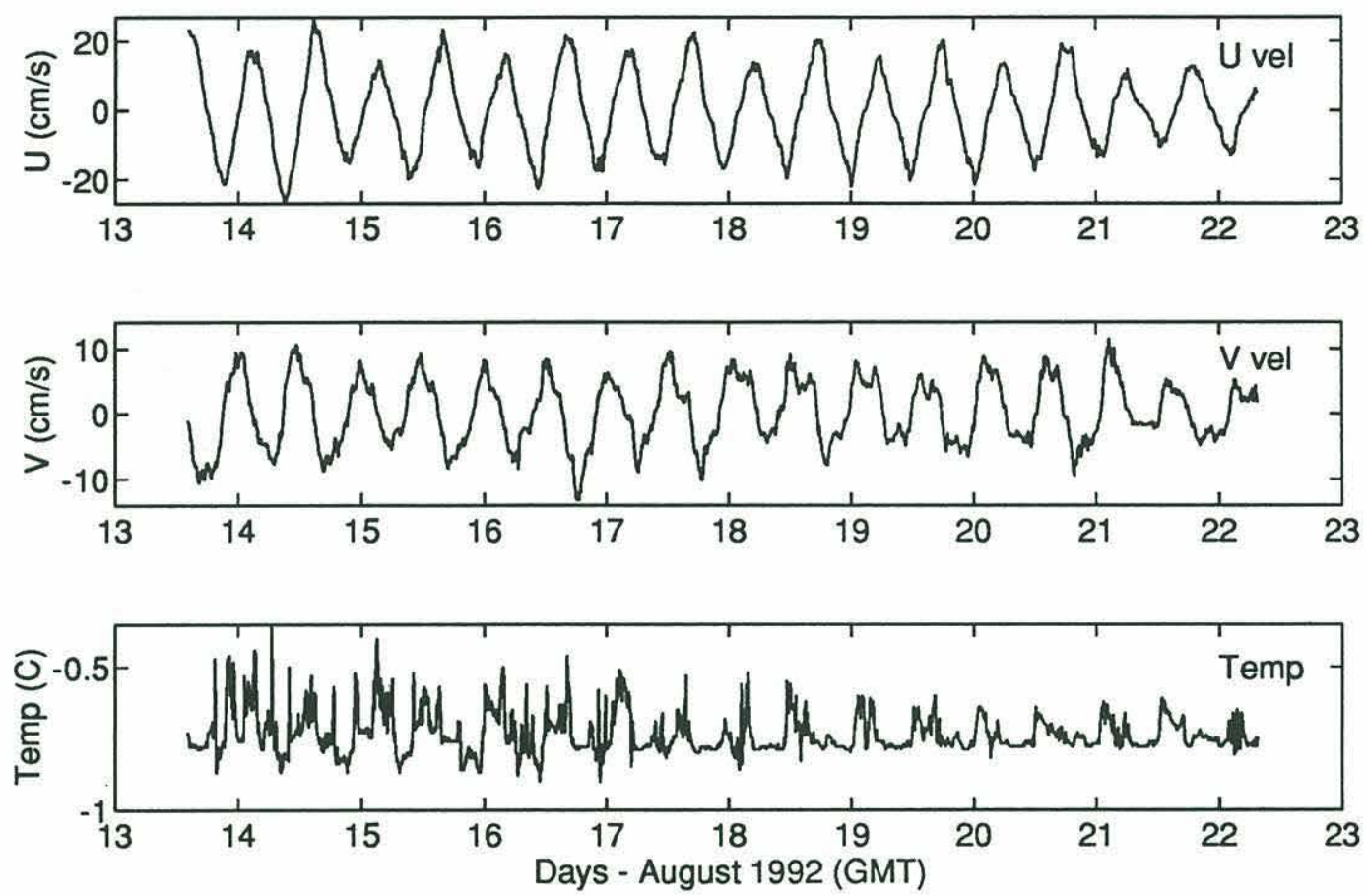

Figure 4-4: $U$ and $V$ velocity, and Temperature records from Aanderaa \#9259 at the $\mathrm{NE}$ array at a depth of 82.1 meters.

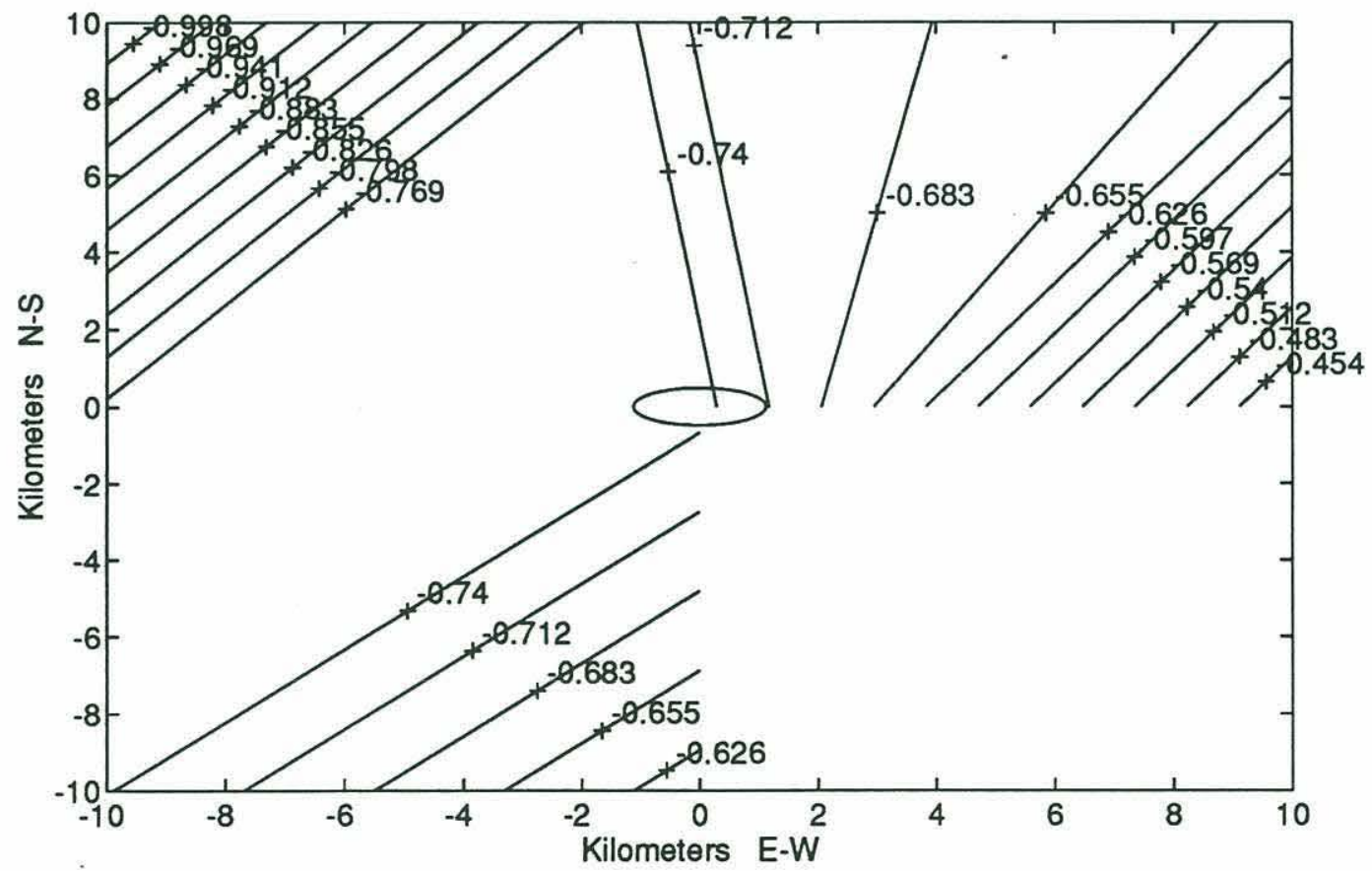

Figure 4-5: Temperature contour map at NE mooring at a depth of $82.1 \mathrm{~m}$ using adjacent CTD stations. The current ellipse from Aanderaa \# 9259 shows the amount of horizontal advection during one tidal cycle. 
temperature by 90 degrees, at the time when the current shifts from flowing westward to flowing eastward, the temperature will be at a maximum, i.e. it is at this time that the warm water to the east will have been advected the greatest distance to the west. This can also be seen by taking the integral of the $u$ velocity; the displacement record is now 180 degrees out of phase with the temperature record which is consistent with having the warmer water to the east.

The displacement ellipse was then plotted over the temperature contour. From this plot, one sees that the maximum change in temperature due to horizontal advection is $0.037{ }^{\circ} \mathrm{C}$, which only accounts for 18 percent of the peak to peak temperature change. Another argument against horizontal advection being the mechanism behind the temperature changes is that the $-0.9^{\circ} \mathrm{C}$ water would have had to been advected from over $14 \mathrm{~km}$ away from the northwest and the $-0.36{ }^{\circ} \mathrm{C}$ water would have had to been advected from over $10 \mathrm{~km}$ away from the east or over $20 \mathrm{~km}$ away from the south. None of these distances are credible given the results listed in Table 4.1. Analyses of

Table 4.1: Horizontal Advection of Water due to Barotropic Tides

\begin{tabular}{|c|c|c|c|}
\hline Array & Depth (m) & $\mathrm{N}-\mathrm{S} \operatorname{disp}(\mathrm{km})$ & $\mathrm{E}-\mathrm{W} \operatorname{disp}(\mathrm{km})$ \\
\hline$\overline{\mathrm{NE}}$ & 82.1 & 0.98 & $\overline{2.2}$ \\
\hline NW & 49.3 & 1.76 & 2.46 \\
\hline SW & 80.0 & 0.89 & 1.82 \\
\hline
\end{tabular}

the SW and NW moorings yield similar results.

\subsection{The Internal Tide and Internal Wave Spec- trum}

In this section we convert temperature fluctuations into particle displacement fluctuations and then into internal tide and internal wave amplitudes using Fourier analysis. In performing this analysis we assumed that the temperature fluctuations can be used to obtain the magnitude of the particle displacement. This will be true if the temperature-salinity (T-S) relationship is constant, i.e. one can go uniquely from 


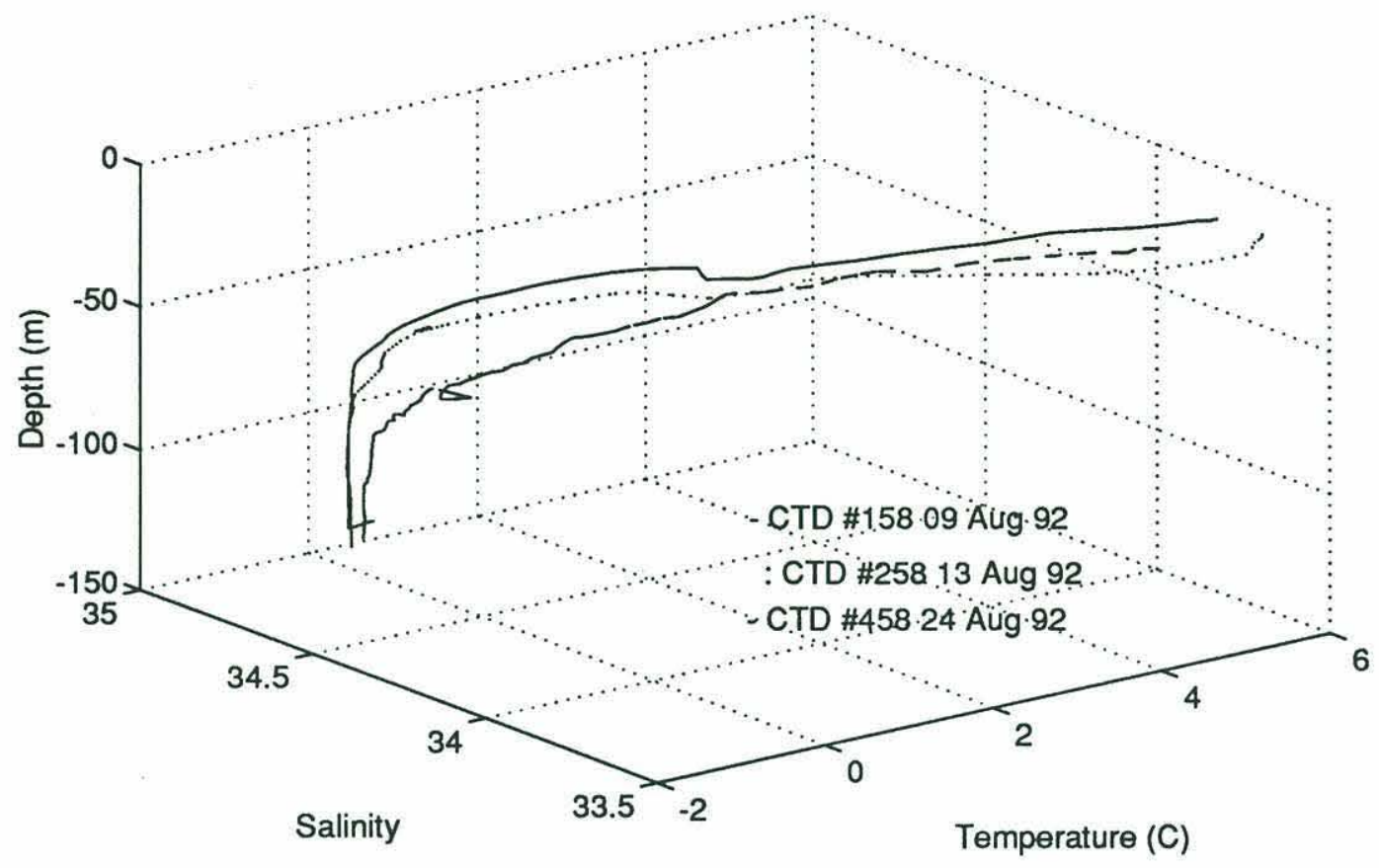

Figure 4-6: Temperature and Salinity versus depth for the CTD casts taken at the NE array.

temperature displacement to density displacement. Figure 4-6 shows the relative constancy of the T-S relationship over three different CTD casts at one site. A rise in temperature for a sensor (see Fig. 4-7) above the mixed layer (i.e. negative temperature gradient) corresponds to a downward displacement of warmer water. The vertical displacement $\delta z$ for a parcel of water is

$$
\delta z=\delta T \frac{d z}{d T}
$$

where $\delta T$ is the temperature fluctuation measured by the Brancker logger, and $d z / d T$ is the temperature gradient at the mooring. We have taken $\mathrm{z}$ as being negative downward. The particle displacement can be determined by integrating (4.1) so that each temperature corresponds to a given depth. This method fails if the temperature profile is double valued near the depth of the logger or the logger is near the knee of the $T(z)$ profile. Using the temperature profiles from the CTD casts, we obtained a best fit temperature profile which preserved the slopes of the CTD temperature profiles. Each temperature record was then converted to a particle displacement 


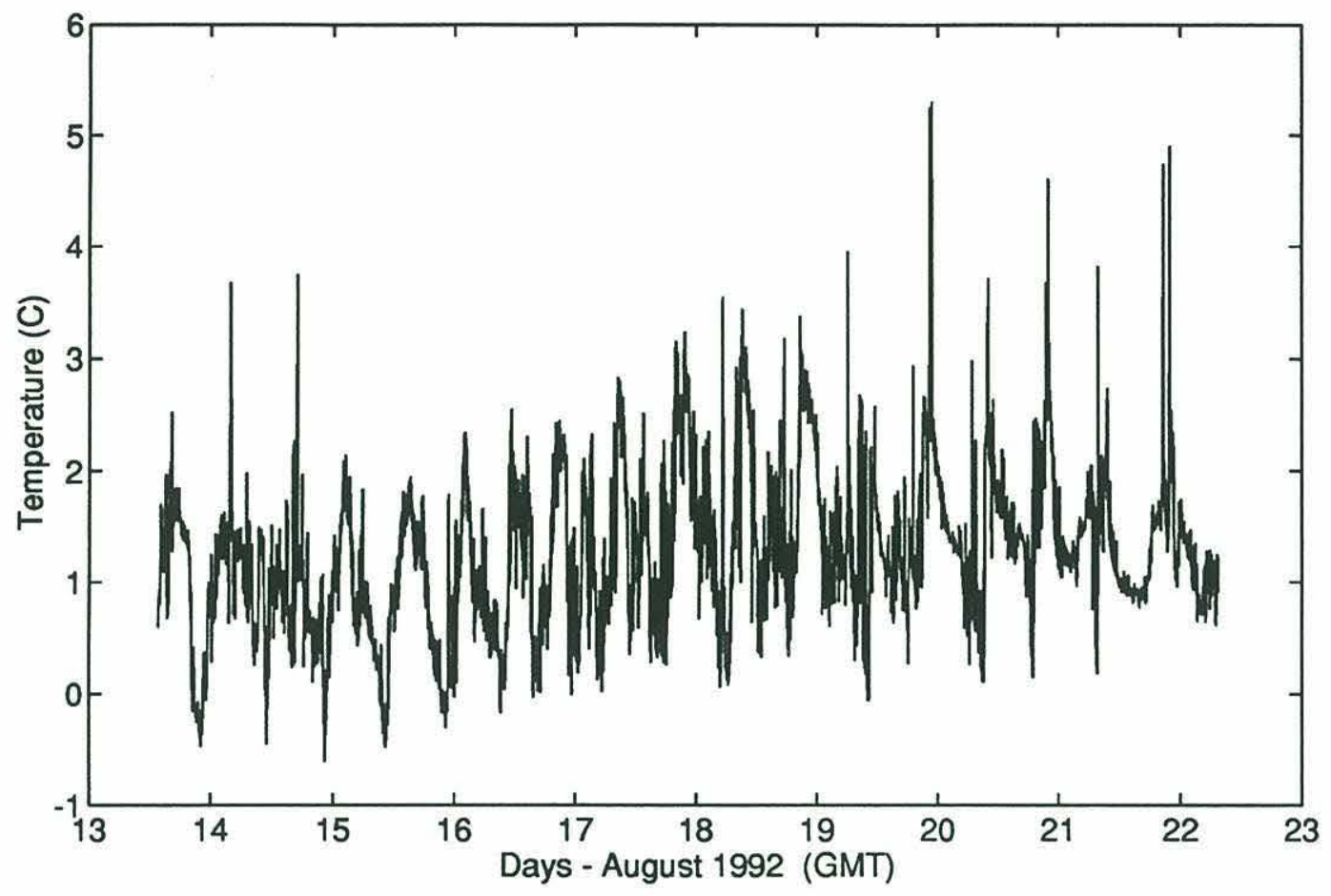

Figure 4-7: Temperature record for Brancker logger \#3663 at NE mooring at a depth of $32.1 \mathrm{~m}$.

record (see Fig. 4-8). The method assumed that the best-fit linearized temperature profile was temporally stationary, whereas in fact it is not quite, as demonstrated by Fig. 4-3. Additionally, in several records the temperature minima and maxima recorded by the Brancker temperature loggers exceeded the temperature minima and maxima recorded during the CTD casts. The CTD casts represent "snapshots" of the temperature profile, and of the ten casts taken at the NE, SW, or NW moorings only two occurred (one at the NW mooring and one at the NE mooring) when the moorings were deployed, and these CTD casts were at the ends of the temperature records. In order to compensate for this mismatch, the temperature profiles were extended at the upper and lower ends while maintaining the same slope. This process was analogous to including some horizontal displacement. It must be remembered that there are two processes taking place, horizontal advection and vertical displacement, and that when the Brancker temperature exceeds the CTD temperature, what has occurred is obviously both a horizontal advection and a vertical temperature displacement.

To estimate the error of extending the temperature profiles, the temperature 


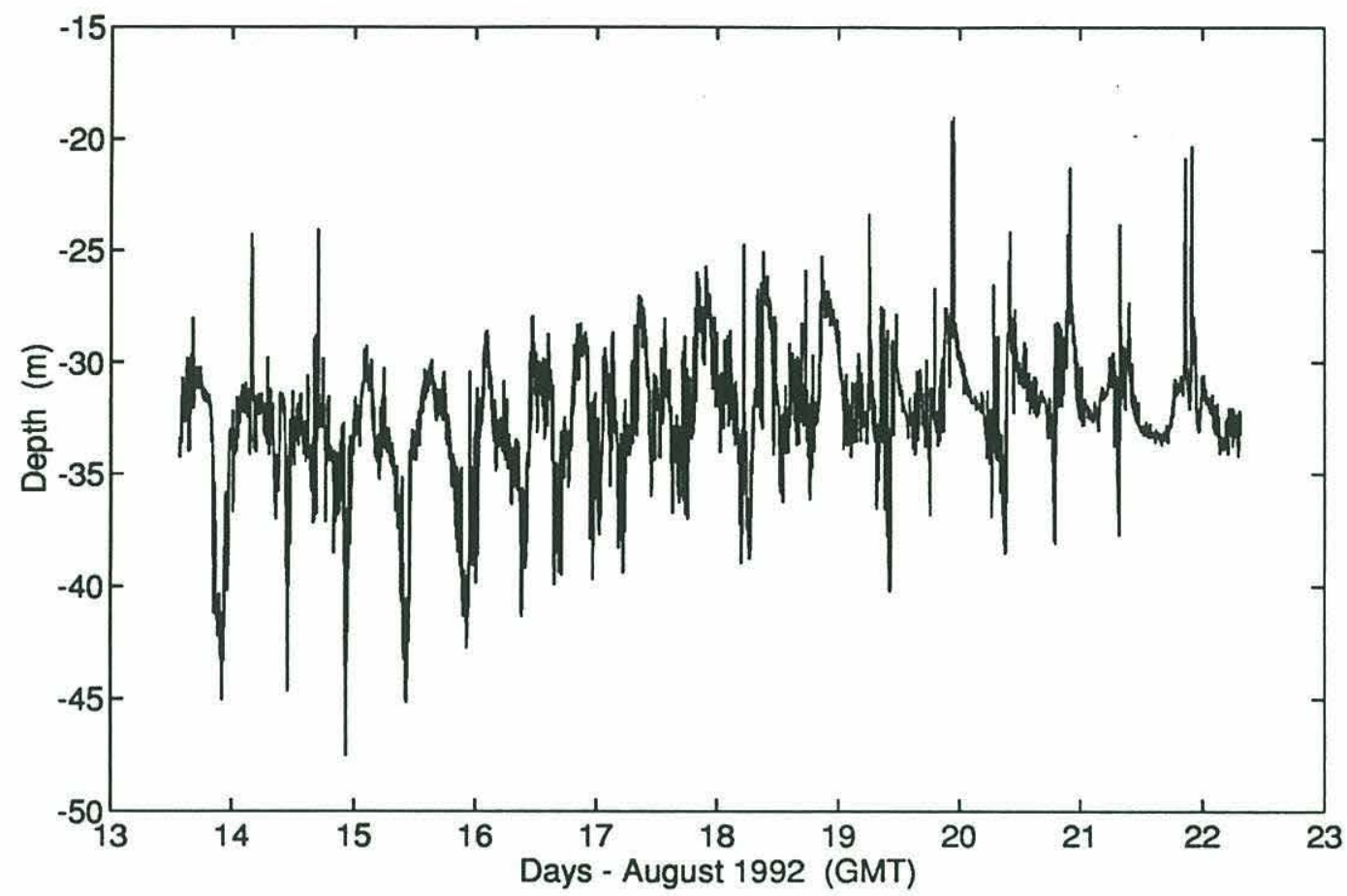

Figure 4-8: Particle displacement $\zeta$ calculated for Brancker logger \# 3663 on NE array at a depth of $32.1 \mathrm{~m}$.

record for Brancker \#3660 was examined. This temperature record, like the others at the SW mooring, showed several eddies, which act similar to a horizontal advection of the profile (e.g. Fig. (4-1). The particle displacement and amplitudes were computed two ways for the Brancker \#3660 temperature record: first, assuming that the eddy was a horizontal advection and adjusting the temperature profile accordingly; and second, filtering out the eddy feature and using an unadjusted temperature profile. The difference between the amplitudes in the internal tide band computed the two different ways was at most less than $3.5 \%$.

Once the particle displacement was computed, it was then Hanning windowed, and analyzed using the Welch method of fast Fourier transform(FFT) spectrum analysis. Figure 4-9 is a typical spectrum. The most significant peak in the internal tide region is the semidiurnal peak. The amplitude was extracted by summing the area under the peak

$$
A=\left[\frac{2}{N} \sum_{k=a}^{k=b} P D(k)\right]^{\frac{1}{2}}
$$




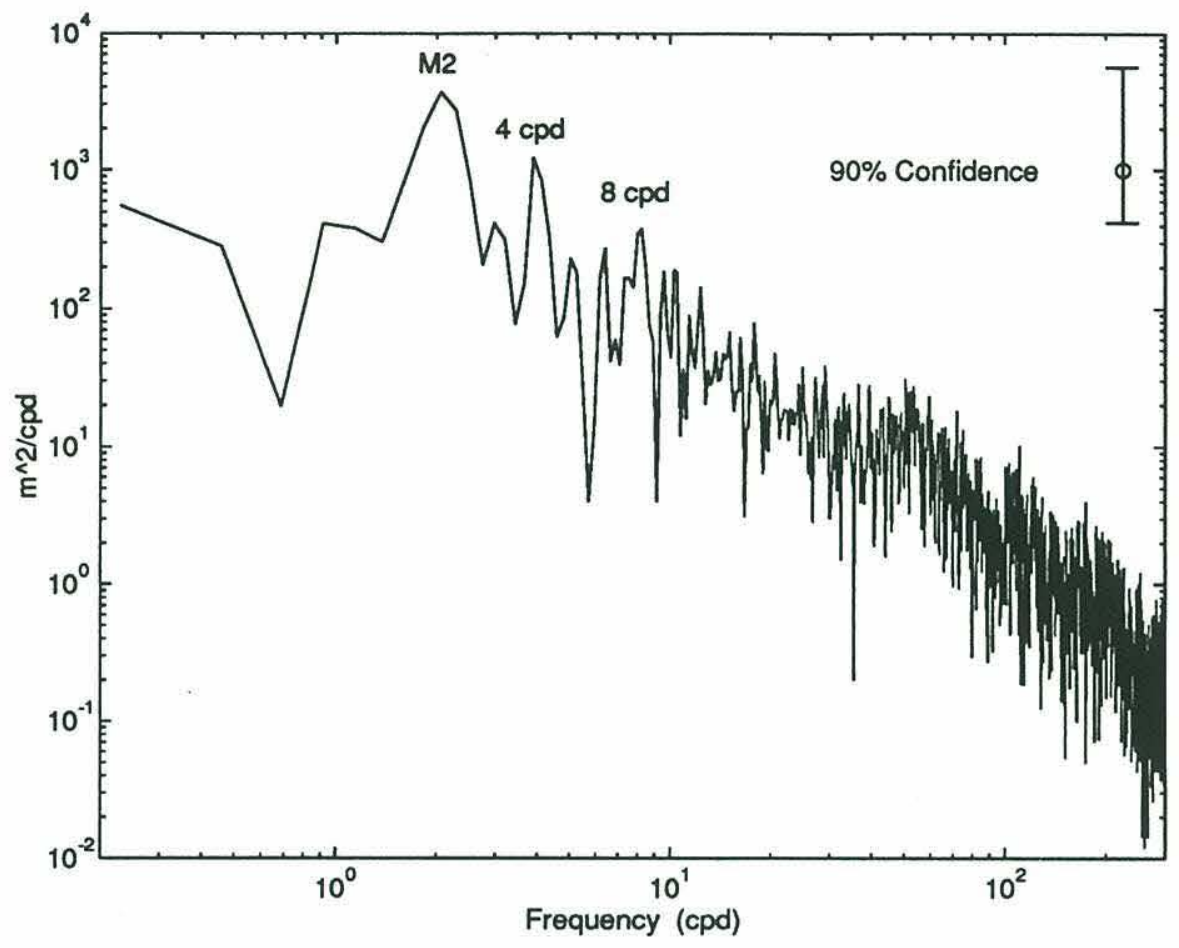

Figure 4-9: Power spectrum density for the particle displacement calculated for Brancker logger \# 3663 on the NE array at a depth of $32.1 \mathrm{~m}$. The estimate has four degrees of freedom.

where $A$ is the amplitude, $P D(k)$ is the power density function at $k$ and $N$ is the length of the sequence. Table 4.2 gives the amplitudes at the Southwest array computed using (4.2) for the M2 tidal component.

Due to the shortness of the records, high resolution Fourier analysis is not possible. This results in combined tidal amplitudes for some frequency values. The $M 2$ peak probably contains $S 2$ and other semidiurnal constituents, though they are small. Also, the amplitudes computed this way are average amplitudes, and therefore smooth out

Table 4.2: SW Mooring Particle Displacement Amplitudes $\zeta$

\begin{tabular}{|c|c|}
\hline Logger Depth(m) & $\mathrm{M} 2(\mathrm{~m})$ \\
\hline$\overline{29.9}$ & 1.8 \\
\hline 39.9 & 2.1 \\
\hline 140.0 & 7.9 \\
\hline 200.5 & 6.1 \\
\hline
\end{tabular}




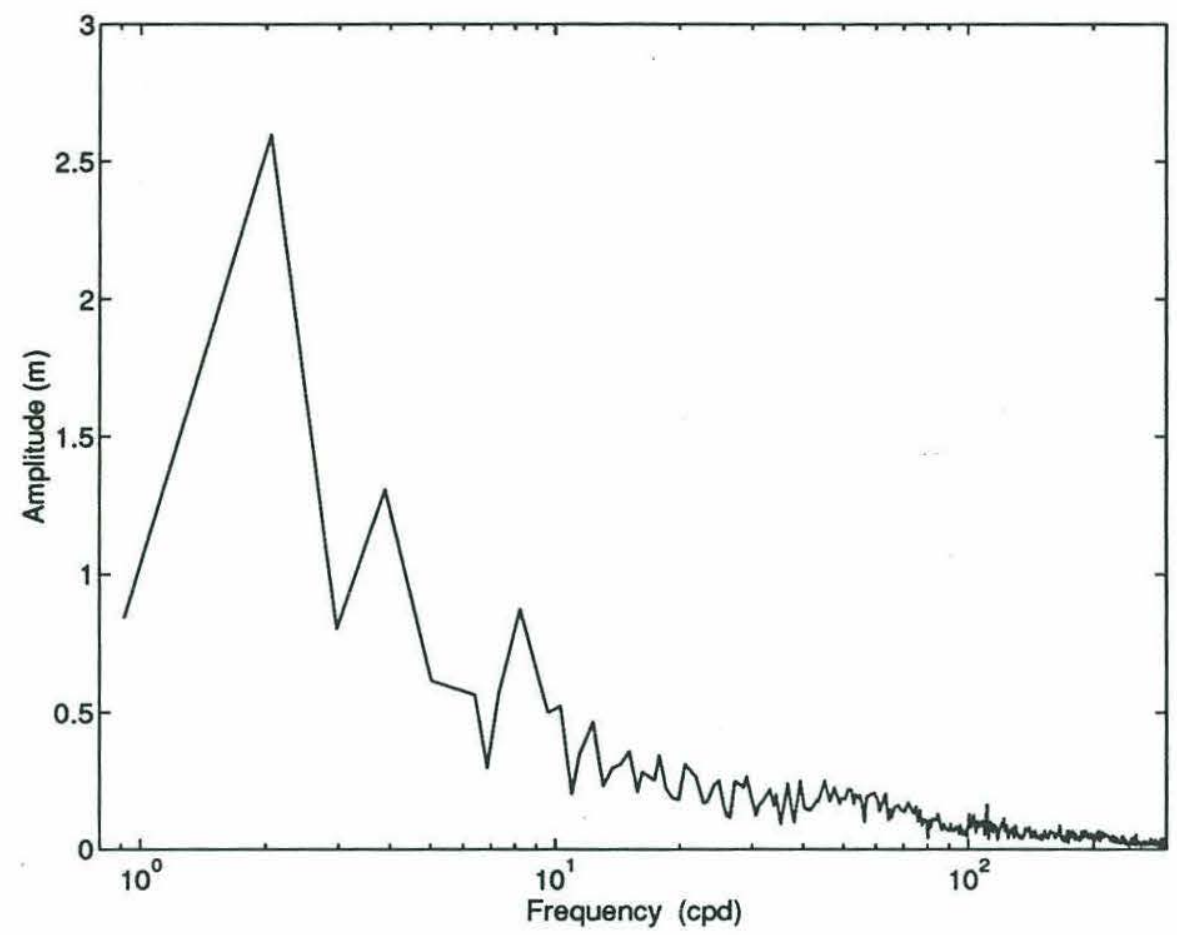

Figure 4-10: Amplitude spectrum for Brancker logger \# 3660 on SW array at a depth of $39.9 \mathrm{~m}$.

the previously noted intermittent nature of the internal tides and internal waves. This method gives reliable estimates for frequencies in the internal tide range (1-10 cpd), but may underestimate the amplitudes in the higher frequency internal wave band. This effect will be addressed when estimating the amplitude spectrum used in equation (2.70). Figure 4-10 shows a typical amplitude spectrum.

The amplitudes extracted using Fourier analysis are used to determine the relative weights of each of the normalized internal tide and internal wave modes. This analysis is performed for the SW mooring because it contains four Brancker temperature loggers and spans $170 \mathrm{~m}$ of the water column, while the NE and NW moorings only have two temperature loggers and span only $10 \mathrm{~m}$ of the water column. Also, using a least squares fit, up to four modes can be estimated at the Southwest array (Fig. 4-11(a)). The least squares fit was simplified by averaging together the two shallow loggers, as they are only $10 \mathrm{~m}$ apart and can be approximated as a single sample. Figure $4-11(b)$ is the result of this average. The results from both methods were consistent. The fit was only performed for frequencies up to $30 \mathrm{cpd}$, since this is 
the maximum buoyancy frequency for the two deep loggers, and in fact, the method appears to break down at about $27 \mathrm{cpd}$. Also, the sign of the mode shapes was consistent in the internal tide band ( $0-8 \mathrm{cpd})$, but the sign of the third mode changed in the frequency band of $10-30 \mathrm{cpd}$. This change coincided with change from the logger at $140 \mathrm{~m}$ having the largest amplitude in the internal tide bank to the logger at $200 \mathrm{~m}$ having the largest amplitude in the internal wave band. The percentages were averaged over the internal tide and internal waves, resulting in an average percentage for each of the first three modes of: mode \#1 -0.58 , mode \#2 - 0.26, and mode \#3 -0.16 . As a check on these numbers, the modes were also estimated incoherently, by performing a least squares fit of the first mode squared to the square of the amplitudes. The residual was then least square fitted to the second mode squared. This process was performed for the first three modes at the $M 2$ tidal frequency. The resulting relative percentages for the first three modes were: mode \#1 - 0.62, mode \#2 - 0.22, and mode \#3-0.16. Both of these sets of numbers are consistent with each other and the results reviewed in Wunsch [1975].

\subsection{Review of Assumptions}

We are now ready to compute the acoustic fluctuations using Eqs. (2.53) and (2.70), but before doing so a review of the validity of the assumptions is warranted. First, we have assumed, in truncating the IW mode series to three modes, that the internal tide and internal wave spectra are red i.e. most of the energy is in the lowest modes. This is probably an excellent assumption, as numerous experiments have concluded that the bulk of the energy in both the internal tide and internal wave bands is in the lowest modes. The ratios of the first three internal wave modes calculated in Section 4.2 show good agreement with those in Wunsch [1975].

Second, we have assumed in solving equation (2.70) that the direction of propagation is along-shelf instead of across-shelf. We assumed this direction of propagation for two reasons: first, we didn't have available with our single point moorings any information on the direction of propagation; and second, this direction $\left(\theta_{0}=\pi / 2\right)$ 

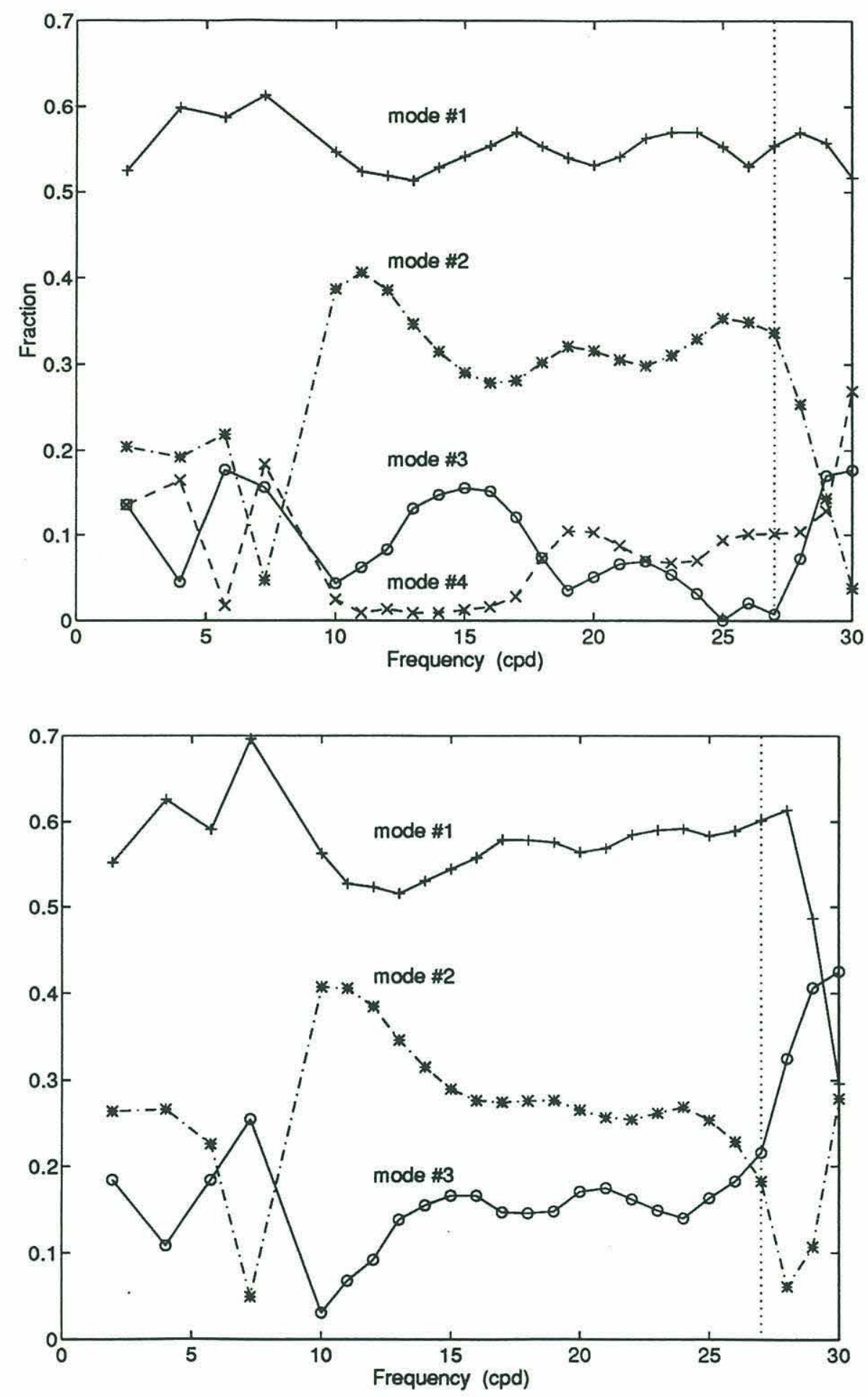

Figure 4-11: (a) is the least squares fit of four amplitudes measured at the SW array to a sum of the first four IW modes. (b) is the same calculation except that the two shallow Branckers are averaged together. In both figures, considerable noise is evident in the internal tide region, and the method is unreliable above about $27 \mathrm{cpd}$ 
maximizes the $\sin c^{2}$ function and thereby, the travel time perturbation covariance (Eq. 2.61). This gives the largest effect we could hope to see, and thus represents an "upper limit" calculation. Additionally, although the assumption of along-shelf propagation is more tenuous than that of across-shelf propagation, it is perhaps not wholly unjustified, since the experimental region is on the edge of the Bear Island trough and in the vicinity of the strong Barents Sea Polar front. As discussed in Section 2.2.2, although internal tides and internal waves propagate shoreward, one experiment noted that the internal tides propagated along the coast. It was postulated that either coastal fronts, or mesoscale structures were refracting the internal tide or that internal tides radiating from differently oriented shelves were causing a complicated wave structure [Levine and Richman, 1989]. The experimental area contains both such features. The Bear Island trough certainly qualifies as a shelf area with different orientations.

Third, we have assumed that the change in temperature seen by the logger was largely due to a vertical particle displacement caused by internal tides and internal waves rather than due to a horizontal advection caused by the barotropic tide. We showed in Section 4.1 that at most $18 \%$ of the change in temperature could be attributed to horizontal advection. This effect was included implicitly in determining the particle displacements, but by not including it resulted in a less than $3.5 \%$ error.

Fourth, we have assumed that $\delta C(\mathbf{x}, t) \gg \mathbf{u}(\mathbf{x}, t) \cdot \tau$. From the Aanderaa data the maximum current was $0.325 \mathrm{~m} / \mathrm{s}$, whereas in the mixed layer, a $5 \mathrm{~m}$ particle displacement could result in a $6.0 \mathrm{~m} / \mathrm{s}$ change in sound speed. 


\section{Chapter 5}

\section{Travel Time Disturbance Predictions}

Using the theory from chapter two, and the internal wave frequency spectrum and amplitudes generated using the methods of chapter four, a modal picture and a ray picture of the travel time perturbations are now generated. These predictions will eventually be compared with the actual results from the experiment, when these become available from colleagues of the Naval Postgraduate School who are working on the travel time estimation problem.

\subsection{Theoretical Results for the M2 Tide}

In chapter two, a formulation for the travel time perturbation, equation (2.53), and travel time covariance, equation (2.70), was derived, using adiabatic normal modes as . a basis. The adiabatic assumption requires a "slowly" varying sound speed profile and bathymetry. We further assumed range independence for the group velocity dispersion $G_{m}(z)$ and for the particle displacement $\zeta$. In evaluating equations (2.53) and (2.70), we chose a simple, two-segment, acoustic and oceanographic model. The model was generated by dividing the acoustic path from the Northeast mooring to the Southeast mooring into two, flat-bottomed segments. Segment number 1 is north of the BSPF, while segment number 2 is south of the front. Each segment's depth is the average 


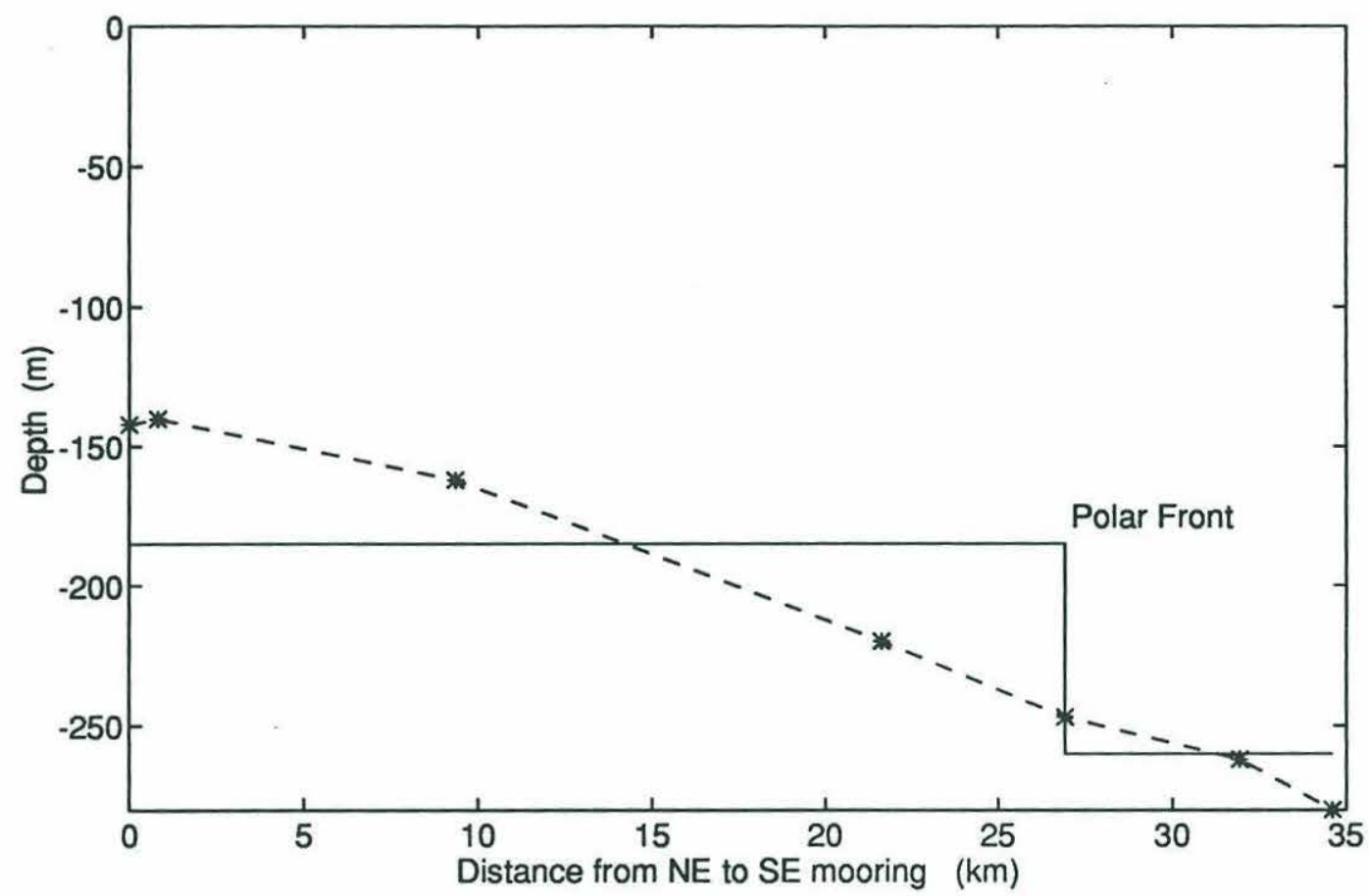

Figure 5-1: Bathymetry of two segment range dependent model from NE array to SE array. Solid line is the depth of the model. Dashed line is the depth at each CTD station. Segment \#1 goes from $0-26.93 \mathrm{~km}$.

of the depths recorded at each of the CTD stations between the moorings. Figure 5.1 shows the bathymetry (using CTD station depths) superimposed on the segment depths. The physical properties (temperature, salinity, et al.) are the averages from CTD stations between the moorings. Figures 5-2(a) and (b) show the average sound profiles of each segment.

The total travel time disturbance will be taken as the sum of the disturbances in the two segments. This is somewhat inconsistent in that it ignores mode coupling effects due to the front. However, the exact magnitude of the mode coupling due to the front is not well known at this time, so we did not pursue this correction to our result.

\subsubsection{Acoustic Ray Results}

The simplest, shallow ocean model, given a strong mixed layer, is a two-layer ocean. In this model, the sound speed of each layer is the vertical average of the Fig. 5-2 

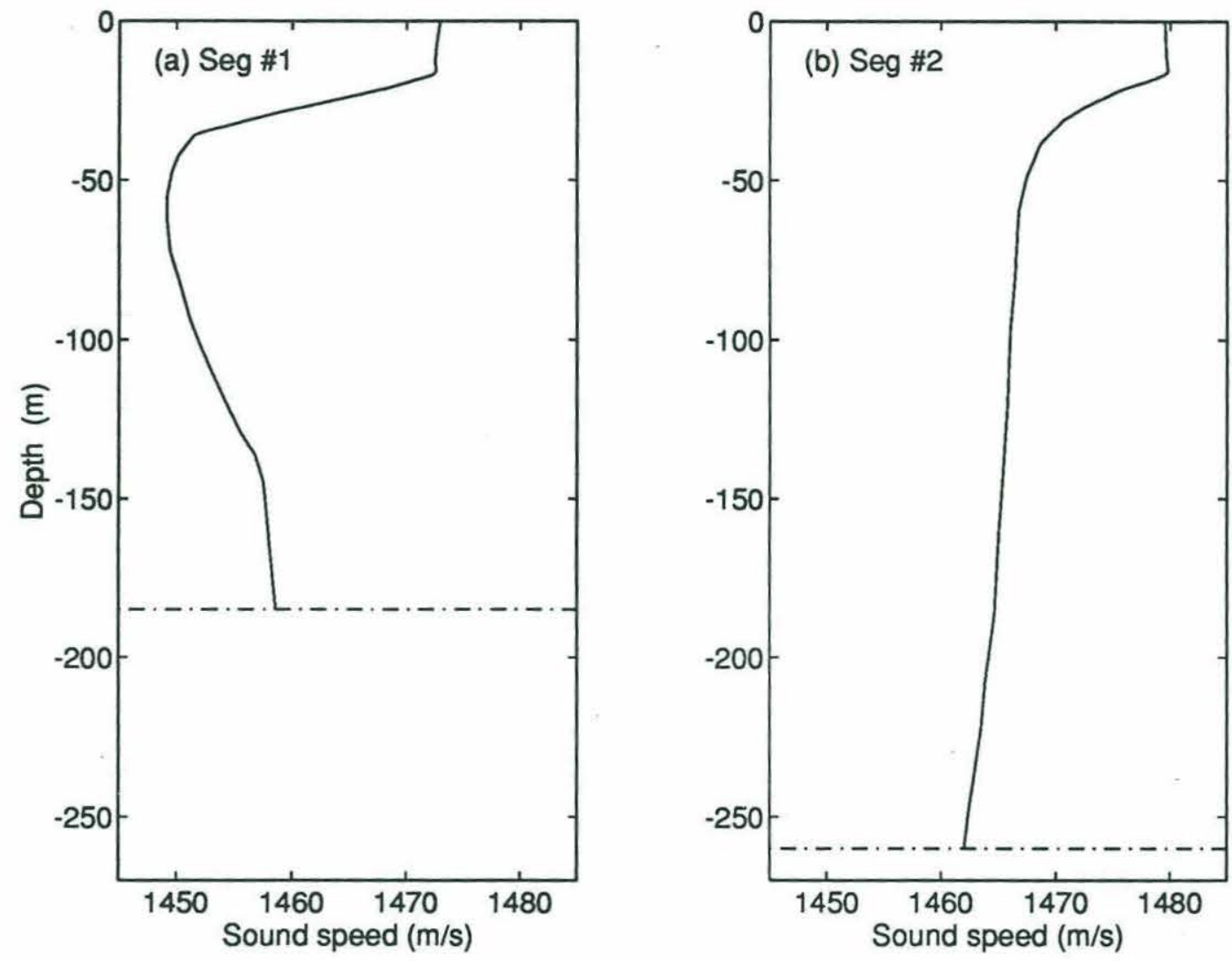

Figure 5-2: (a) Averaged sound speed profile for segment \# 1, and (b) Averaged sound speed profile for segment \# 2

sound speed profile for a given range segment. Table 5.1 lists the details for each segment.

The travel time perturbations in ray theory can easily be calculated by perturbing the layer an amount equal to the peak to peak particle displacement $\zeta$ and input ting the resultant sound speed perturbation $\delta C$ into a discretized form of equation (2.7). This gives

$$
\delta T=\sum_{n=0}^{h} \frac{2 \delta C\left(z_{n}\right) N \triangle z}{C_{0}^{2} \sin \chi\left(z_{n}\right)}
$$

where $N$ is the number of complete ray cycles, and $\chi$ is the grazing angle.

This calculation was run on segment \# 1 for the M2 internal tide using a peakto-peak particle displacement $\zeta$ of $11 \mathrm{~m}$ with a source and receiver in the lower 1 aver. It was assumed to first order that neither $\chi$ nor $N$ changed, that is the ray pat $\mathrm{b}$ was identical before and after the sound speed profile was perturbed. Figure 5-3(a) shows 
the results for eigenrays which penetrate the upper (mixed) layer.

Figure 5-3(b) is the calculation performed for segment \# 2 with a peak-to-peak particle displacement $\zeta$ of $15 \mathrm{~m}$, which was the maximum observed displacement of the thermocline seen in Fig. 4-3. The displacement of the thermocline due to the $M 2$ internal tide may be as small as $4 \mathrm{~m}$, which was the computed $M 2$ internal tide amplitude at the SW array using Fourier analysis. Using the smaller value of $4 \mathrm{~m}$ would scale Fig. 5-3(b) by one fourth.

The calculation can also be performed using a more sophisticated ray tracing program such as MPP with fully range dependent profiles and geometry. As we are only interested in order of magnitude estimates with this ray calculation, we have not pursued this.

\subsubsection{Acoustic Normal Mode Results}

In calculating the acoustic modal results, the same two-segment view of the acoustic path was taken. Figures 5-4(a) and (b) show buoyancy frequency profiles calculated from equation (2.25) using averaged temperature and salinity profiles for segments \# 1 and \#2.

Using these buoyancy frequency profiles, equation (2.27) can be solved to give the internal tide and internal wave modes. Figures 5-5(a) and (b) show the first three internal tide modes for each segment at the M2 frequency. Similarly, given the sound speed profiles, equation (2.41) gives the adiabatic normal modes (Figs. 5-6(a) and (b)). In comparing Fig. 5-5 with Fig. 5-6, the case can be made that the third $M 2$ internal tide mode will possibly have the greatest effect on the acoustics, because its

Table 5.1: Two-layer, range independent model

Distance (m)

Avg. water depth (m)

Mixed layer depth (m)

Sound speed $(\mathrm{m} / \mathrm{s})$ upper layer

Sound speed $(\mathrm{m} / \mathrm{s})$ lower layer $\frac{\text { Segment Nr. } 1}{26,930} \quad \frac{\text { Segment Nr. } 2}{7680}$

185

25

1473

1453
260

1479.9

1465.1 

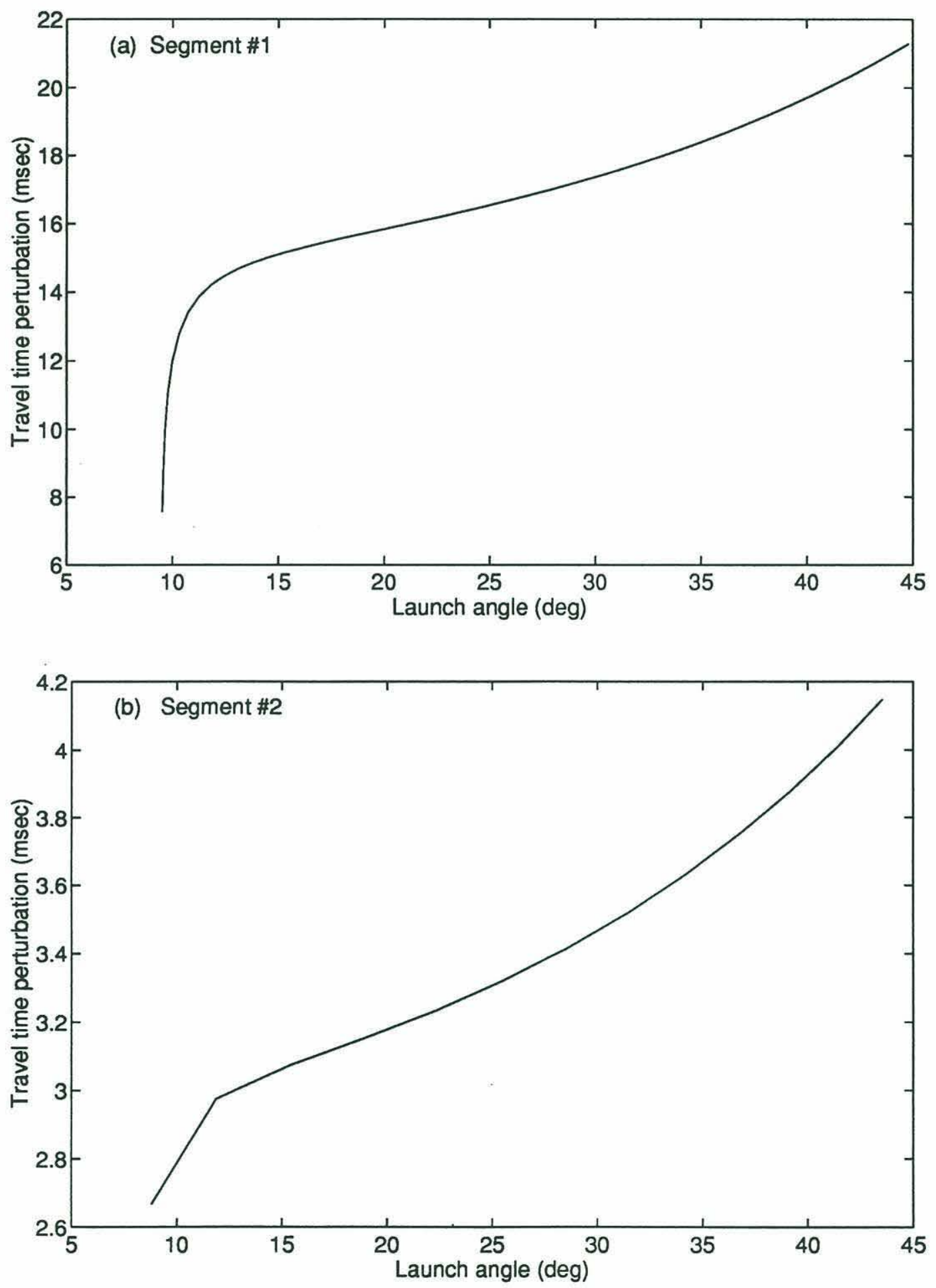

Figure 5-3: Travel time perturbation of eigenrays versus launch angle. 

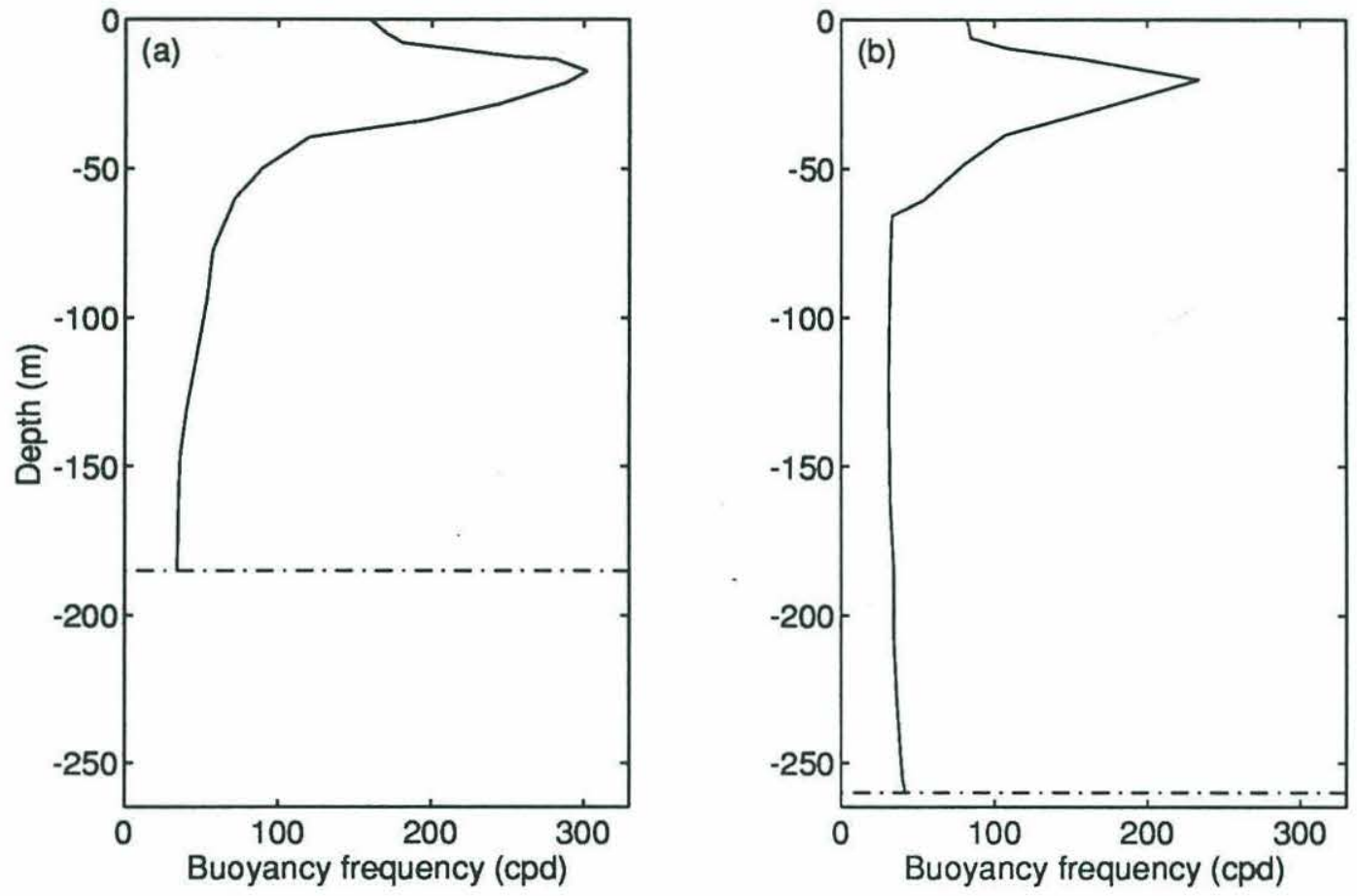

Figure 5-4: Smoothed buoyancy frequency $N$ profiles for (a) segment \# 1 and (b) segment \# 2
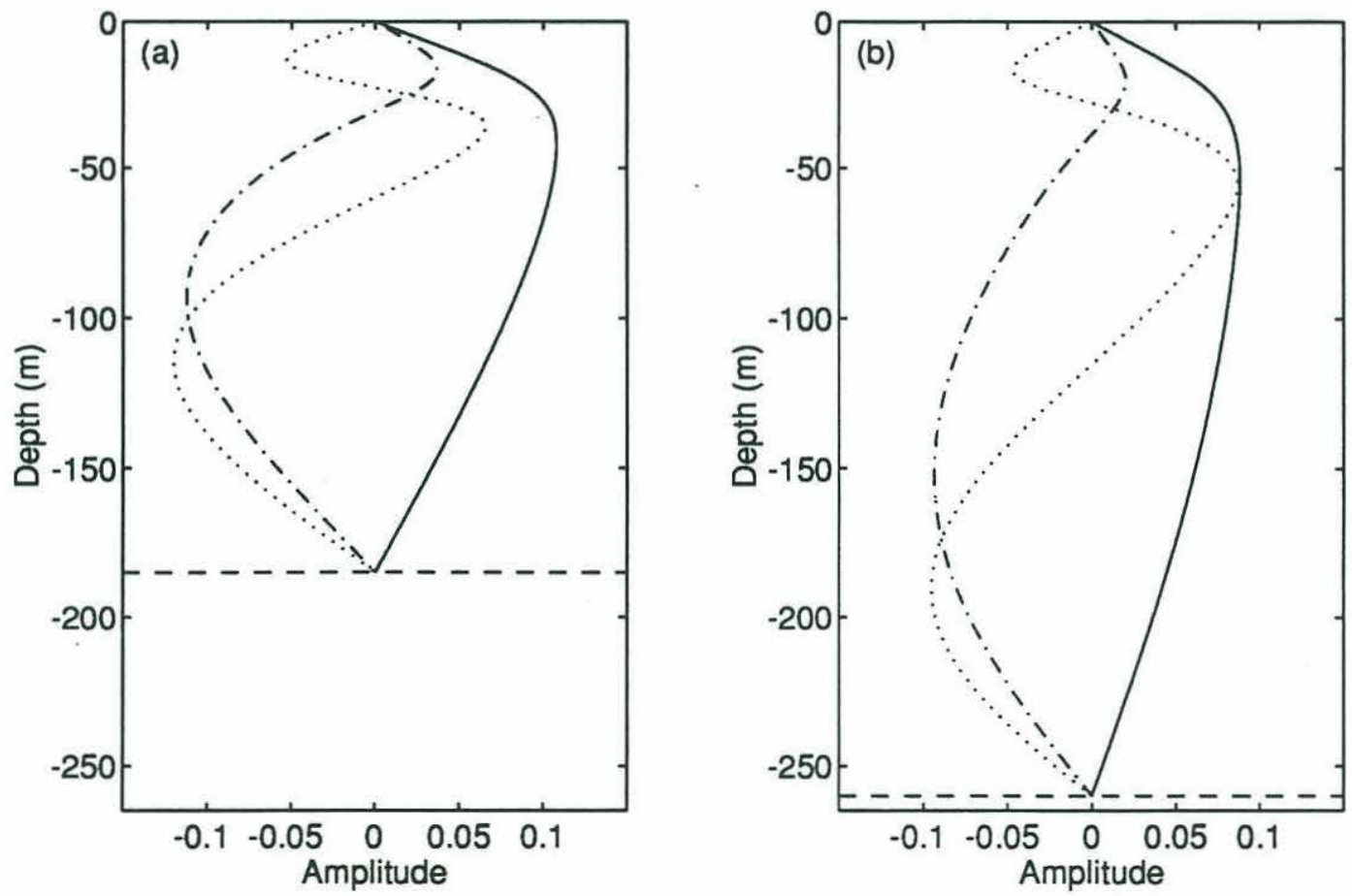

Figure 5-5: First three internal tide modes for (a) segment \# 1 and (b) segment \# 2 at $\omega=M 2$ 

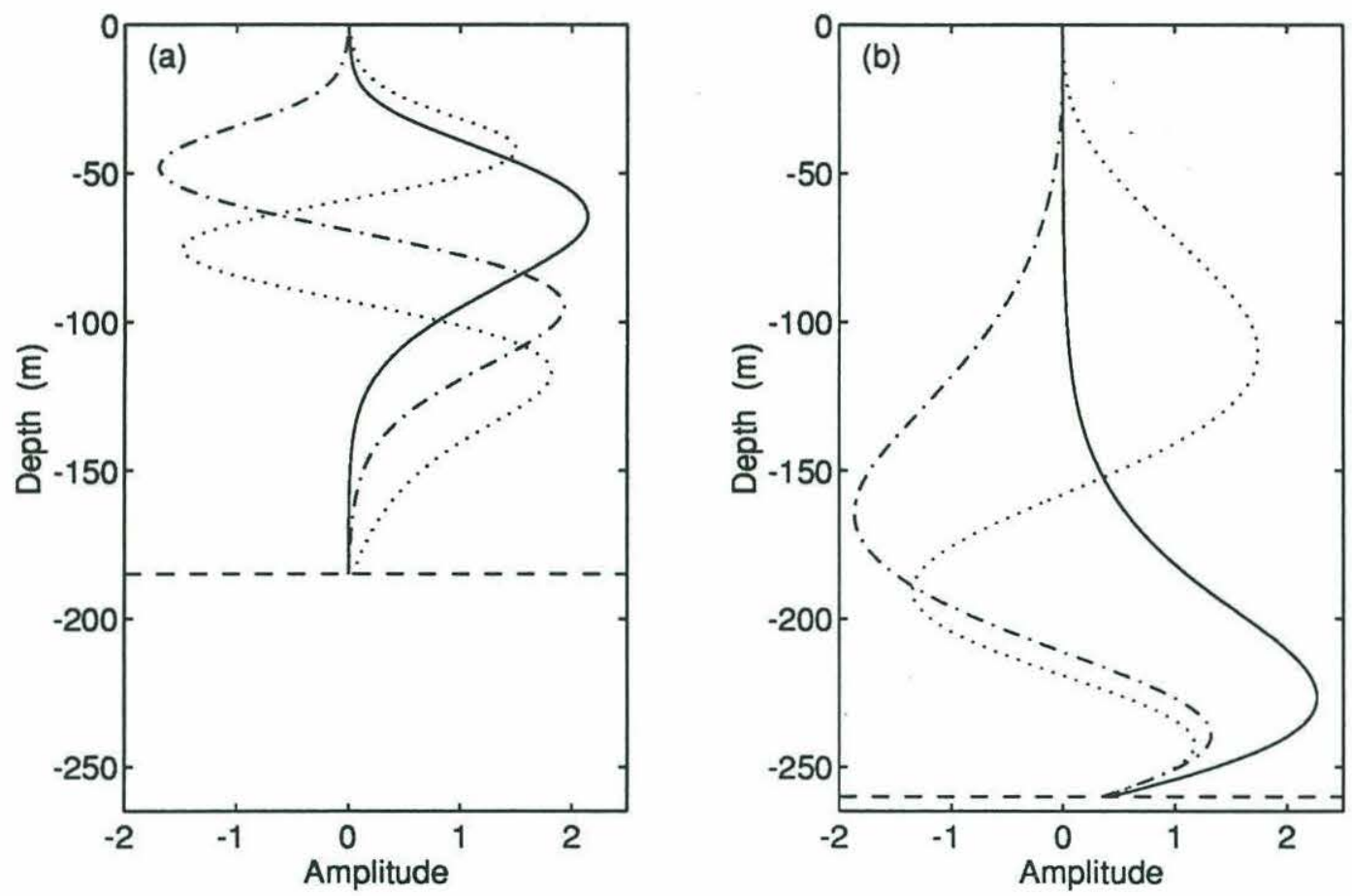

Figure 5-6: First three adiabatic normal modes for (a) segment \# 1 and (b) segment \#2.

maximum occurs near the maximum of the acoustic modes.

The results of calculations using equation (2.53) are shown in Figs. 5-7 to 5-9 in three different ways. First, as shown in Fig. 5-7, we assume that the lowest ten internal tide modes have an equal amplitude of $5.2 \mathrm{~m}$. This calculation allows us to see how the internal tide and acoustic modes will interact, everything else being equal. For segment \#1, the maximum travel time perturbation, indeed, occurs for the third internal tide mode. Except for the first internal tide mode, low internal tide modes dominate the scattering. The internal tide modes have little effect on the first acoustic mode. For segment \#2, the low internal tide modes, including the first internal tide mode, also dominate the scattering, but the first acoustic mode is somewhat more affected by the internal tides. It should be noted that one reason that the travel time perturbations for segment \# 2 are much smaller than those for segment \# 1 is that the range for the former is four times smaller than the latter.

For the second method, shown in Fig. 5-8, only the three lowest internal tide modes are used, instead of using the lowest ten modes as before. The three internal 

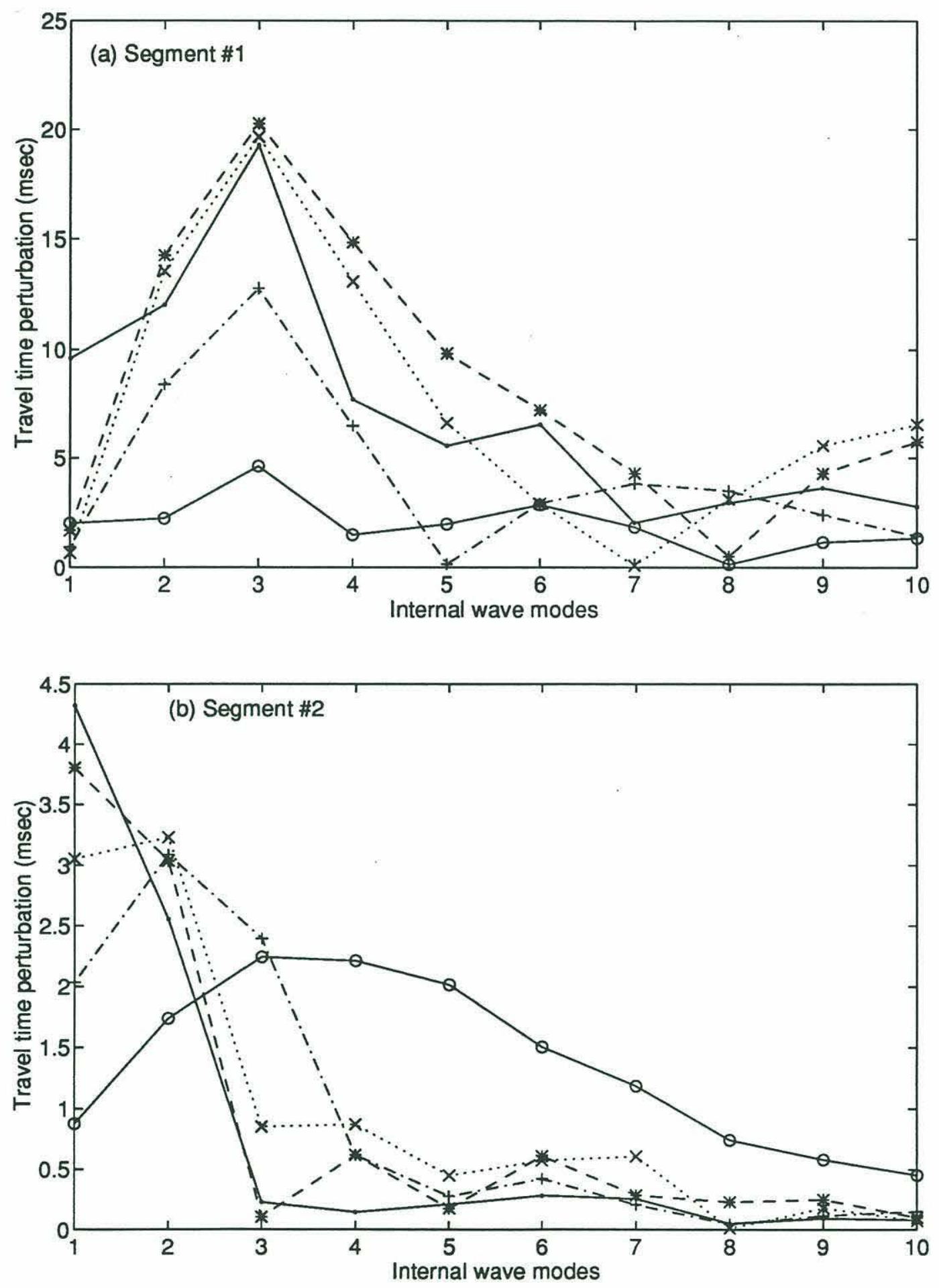

Figure 5-7: Travel time perturbations at $M 2$ using first ten internal tide modes equally weighted with a maximum amplitude of $5.2 \mathrm{~m}$. Lines are acoustic modes: $\# 1(\circ), \# 2(+), \# 3(\times), \# 4(*), \# 5(\bullet)$. 
tide modes are no longer equally weighted, but are now weighted by the amplitude factors, $0.58,0.26$, and 0.16 , respectively, which were calculated in Section 4.2. This calculation allows us to see the contribution of each internal tide mode to the acoustic travel time perturbation. In segment \#1, we see again that first internal tide mode has little effect on the low acoustic modes, and that the low internal tide modes (mode two and three) dominate the low acoustic modes. In segment \#2 the first internal tide mode is dominant for all the acoustic modes calculated.

For the third method, the three lowest internal tide modes are coherently summed together using the signs calculated in Section 4.2. The result is the solid line in Fig. 5-9. The dashed line in Fig. 5-9 is the contribution from the first internal tide mode (the solid line in Fig. 5-8). In both segments \#1 and \#2, the first mode is the dominant contributor to the travel time perturbation. For segment \#1 (Fig. 5-8) the magnitudes of the second and third internal tide modes are greater than that of the first internal tide mode over the range of the first four acoustic modes, but because the signs of the second and third internal tide modes are opposite at depths below 60 $\mathrm{m}$ (where the group velocity dispersion is a maximum at the lower acoustic modes), the effects of the second and third internal tide modes largely cancel each other out, thus making the first internal tide mode the largest component of the travel time perturbation. For segment \#2 this effect is less pronounced, because the magnitudes of the second and third internal tide modes are dissimilar. Figure 5-10 gives the root mean square of the travel time perturbations, the square root of the solution to equation (2.70), for segments \#1 and \#2 using the first three internal tide modes. The rms of the variance of the travel time perturbations for both segments is only slightly smaller than the respective travel time perturbations.

\subsubsection{Comparison of Ray and Modal Results}

For segment \# 1 the Fig. 5-9(a) modal travel time perturbations of 0.5 to 35.5 msecs are of the same order as the ray travel time perturbations of 7.6 to 21.3 msecs. For segment \# 2 the Fig. 5-9(b) modal travel time perturbations of 0.7 to 6.4 msecs are of the same order as the ray travel time perturbations of 2.7 to 4.1 msecs. This gives us 

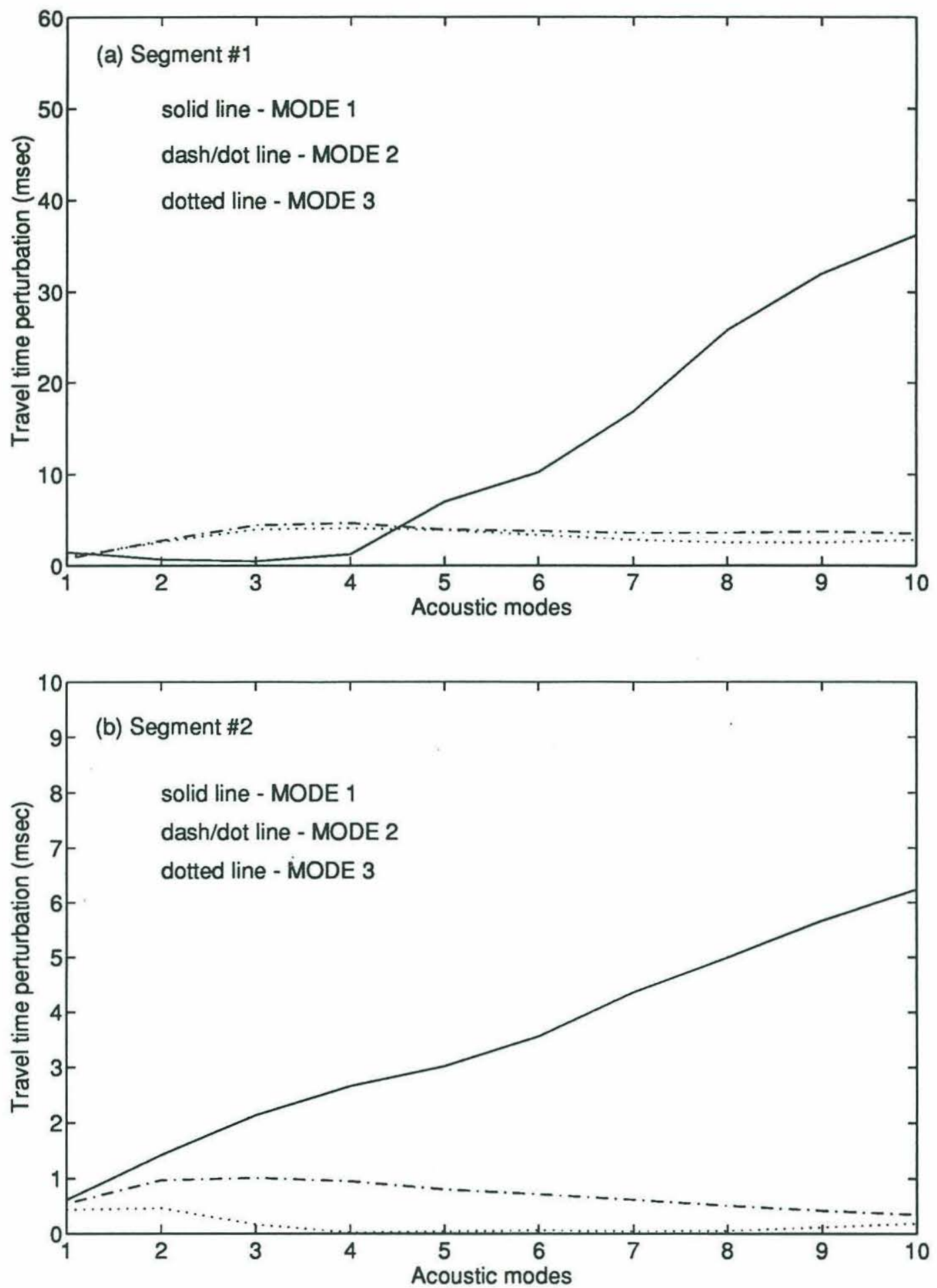

Figure 5-8: Travel time perturbations at $M 2$. The first 3 internal tide modes are shown and are weighted by $0.58,0.26$, and 0.16 , respectively. 

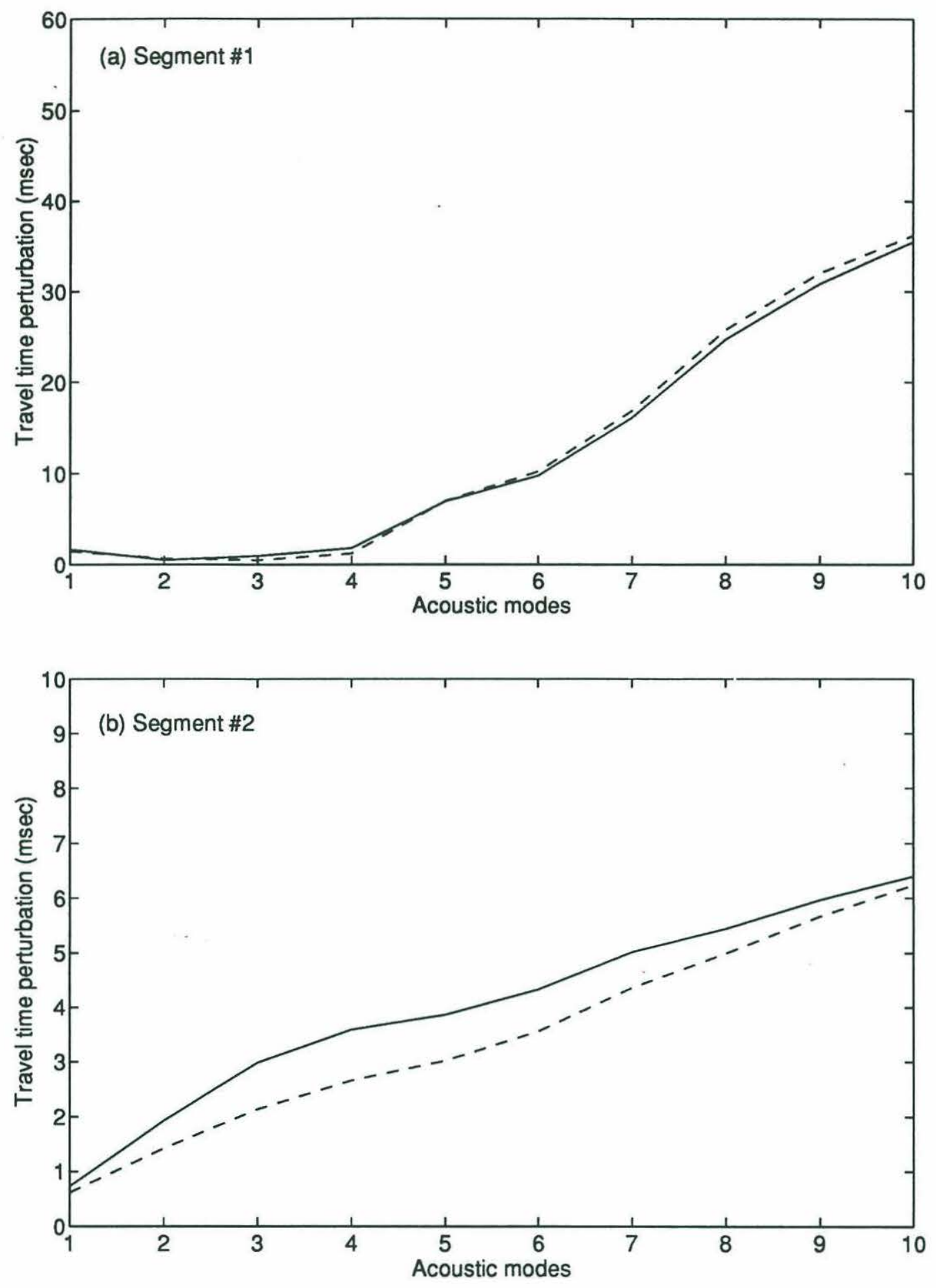

Figure 5-9: Travel time perturbations at $M 2$ using the sum of the first 3 internal tide modes, weighted by $0.58,0.26$, and 0.16 . Shown for comparison (as a dashed line) is the contribution from the first internal tide mode at $M 2$ (Fig. 5-8). 

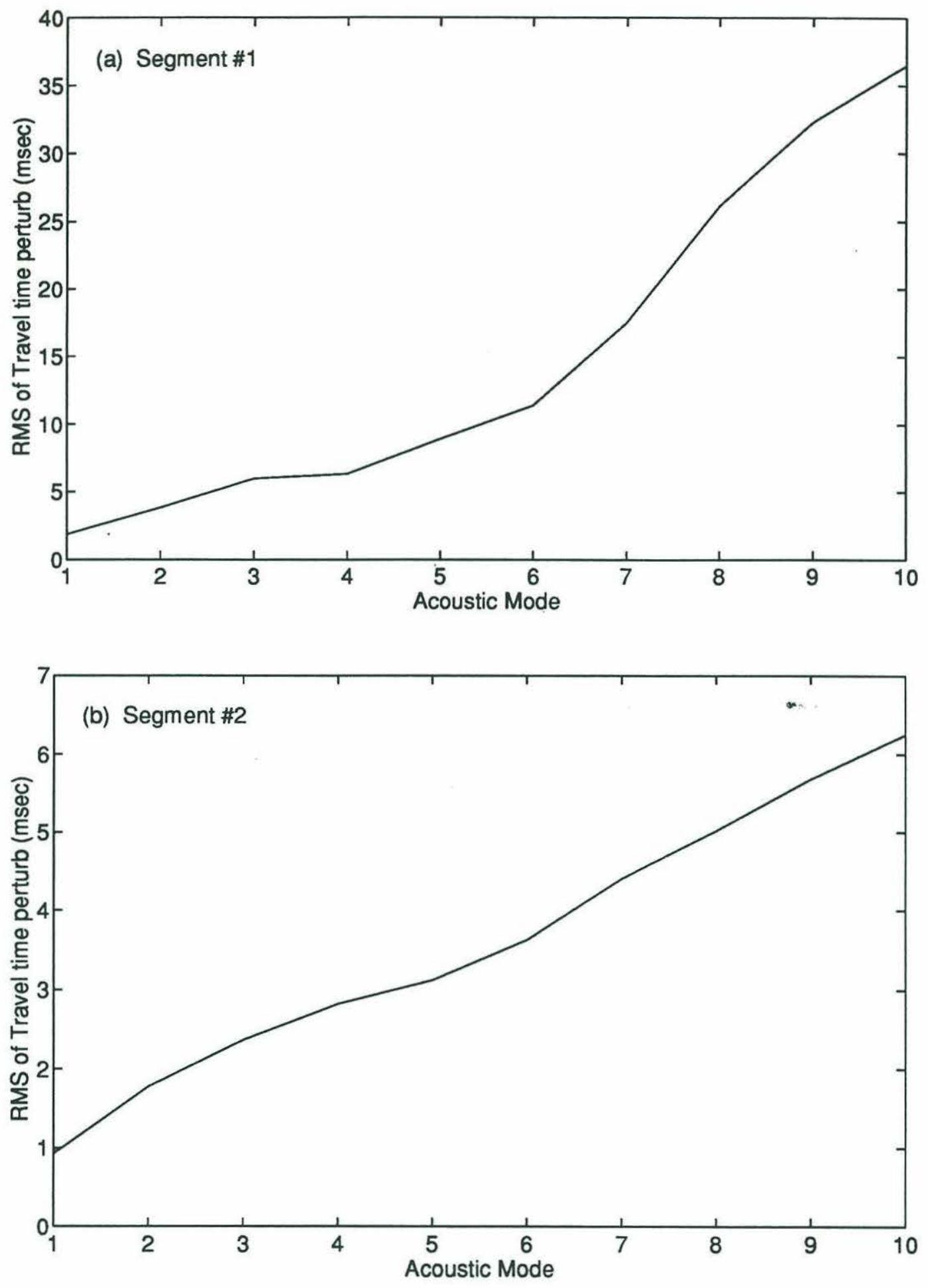

Figure 5-10: RMS of the travel time perturbations at $M 2$ using the first three internal tide modes. 
some confidence that our calculations are at least consistent, within the assumptions we have made.

\subsection{Acoustic Normal Mode Results for the Inter- nal Tide and Internal Wave Fields}

Section 5.1.2 evaluated the travel time perturbations and covariances due to the largest internal tide component, the $M 2$ tide. In this section, using the same two segment model we perform the same calculations over the internal tide and internal wave band. Figures 5-11(a) and (b) are the travel time perturbations over the entire IW band for segments \# 1 and \#2. Although the travel time perturbation over the entire internal wave band has a similar shape to the travel time perturbation from the first IW mode at the M2 tidal frequency (dashed line in Fig. 5-11), the internal wave band contributes significantly to the travel time perturbation. Modal travel time covariances were then computed using equation (2.70). The lowest ten acoustic modes, and the lowest 3 internal tide and internal wave modes were used in the calculations. The equation was discretized and summed from the inertial frequency $f$ to the buoyancy frequency $N$. The calculation is a conservative estimate, in that in the internal tide band, the tidal components were treated as delta functions, and only the $M 2$ tidal component was included. The $4 \mathrm{cpd}$ and $8 \mathrm{cpd}$ components were omitted, as their significance is doubtful (see Fig. 4-9). The internal wave band from $10 \mathrm{cpd}$ to the buoyancy frequency $N$ was treated as a continuous spectrum, and numerically integrated. Figure 5-12 gives the results for the acoustic modes. The rms of the variance of the travel time perturbations for both segments has only increased slightly, when the higher frequencies in the internal wave band are included. This is because the internal wave band is an order of magnitude down compared to the $M 2$ tide in spectral amplitude.

When we look at directional effects on the internal waves and tides, the dominance of the internal tide signal over the internal wave signal becomes even greater. At the $M 2$ tidal frequency, scattering due to waves in the across shelf direction $(\theta=0)$ is only 

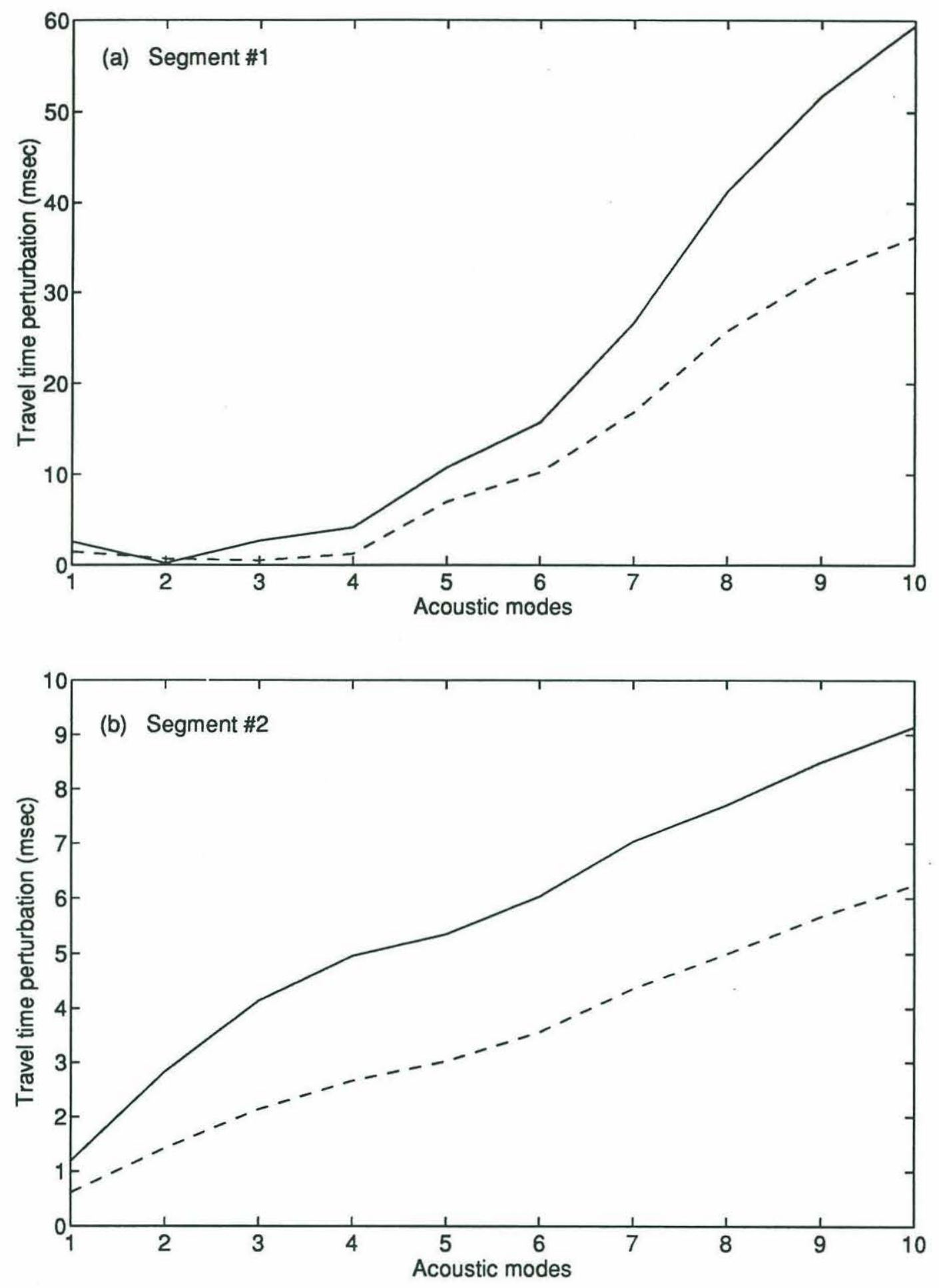

Figure 5-11: Travel time perturbations using the first three IW modes from $f$ to $N$. They are weighted by $0.58,0.26$, and 0.16 . Shown for comparison (as a dashed line) is the contribution from the first internal tide mode at $M 2$ (Fig. 5-8). 

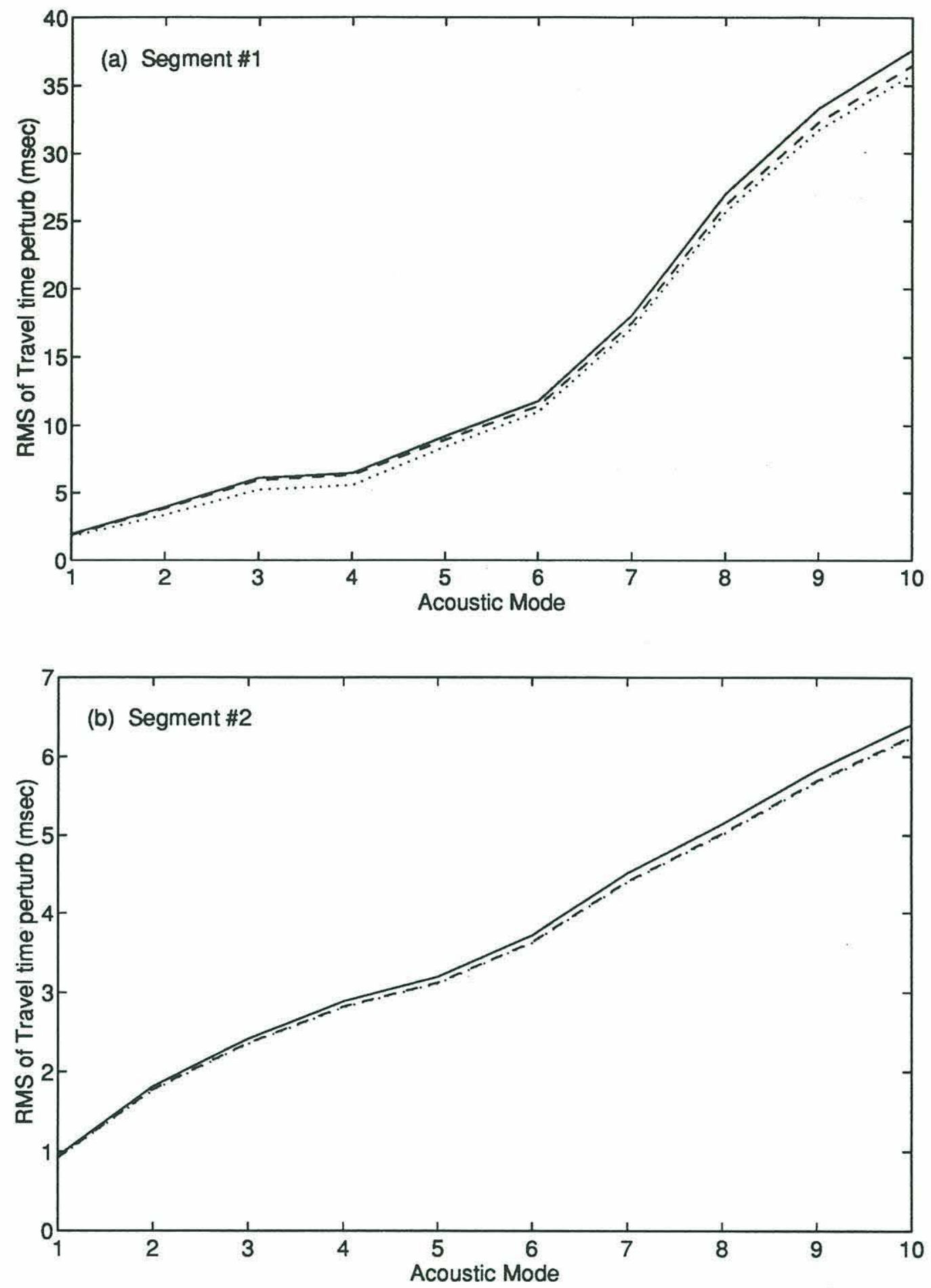

Figure 5-12: RMS of the travel time perturbations, using the first three IW modes, weighted by $0.58,0.26$, and 0.16 , and from $f$ to $N$. The dashed line is the rms of the travel time perturbation for the $M 2$ component. The dotted line is rms of the travel time perturbation for the across shelf case. For segment \#2 the dotted line lies directly over the dashed line. 
slightly less than by waves going along shelf $(\theta=90)$, since for the long wavelength of the first $M 2$ mode $(260 \mathrm{~km})$, the $\operatorname{sinc}^{2}$ function has a value of 0.967 as opposed to 1.0 along shelf. (Thus internal wave scattering is roughly independent of $\theta$ !) For the internal waves, looking at $10 \mathrm{cpd}$ as a representative frequency, the across shelf $\sin c^{2}$ function is 0.0001 (compared to 1.0 for along shelf) due to the higher frequency and smaller wavelength. This compounds the order of magnitude difference in spectral amplitudes.

\subsection{Movement of the Front}

It was pointed out earlier in Section 3.1 that the BSPF can move up to $10 \mathrm{~km}$ over a tidal cycle. To first order, this change can be approximated by taking the differences of the average sound speed between each segment, acting over one kilometer. The result is a travel time perturbation of $4.9 \mathrm{msecs} / \mathrm{km}$, or up to $49 \mathrm{msecs}$ over a tidal cycle. As the acoustic array is not expected to be able to filter out any modes higher than mode \#5, the modal travel time perturbations calculated in Sections 5.1 and 5.2 are not expected to be a significant source of noise for modal inverses for frontal structure. Internal wave noise could be significant, however, for the ray (higher mode) arrivals, a subject needing further investigation. Also, the phase relationship between the perturbation in travel time caused by the internal tide and internal wave fields and the movement of the front is unknown, because of the complex relationships involved in the bathymetry. Finally, due to the fact that one does have a fair degree of horizontal resolution in the cross front acoustic maps made tomographically, we may not be faced with a significant ambiguity between the vertical movement of the internal waves and the horizontal movement of the front. That is, the internal tide/internal wave "noise" may not significantly degrade the maps being made of the front. 


\section{Chapter 6}

\section{Conclusions and Future Work}

As stated in the beginning, our goal in this thesis was to examine using acoustic tomographic techniques to study internal waves and internal tides. Towards that end, we formulated an expression for the travel time perturbation of an acoustic wave by the internal tide and internal wave fields. The perturbation is maximum when the acoustic wave fields and internal wave fields are propagating at right angles to each other. Using temperature records at three deployed moorings, along with the temperature profiles from CTD casts, particle displacement records were obtained. We then determined the internal tide and internal wave spectra using Fourier analysis techniques. The shape of the particle displacement amplitudes at the SW mooring allowed us to estimate the amplitude weights of the first three internal wave modes. Knowing the amplitude spectra we then predicted the acoustic travel time perturbations. We found that for internal wave modes of equal amplitude that the third internal wave mode has the most effect on the acoustic modes. For the $M 2$ tide, the first internal wave mode caused most of the travel time perturbation and variance. When the internal wave band was added, little change from the $M 2$ result was seen for the first five acoustic modes. Since the acoustic VLA is not expected to be able to resolve modes higher than the fifth acoustic mode, for this application the use of the first internal wave mode at the $M 2$ tidal frequency contains the bulk of the travel time perturbation and variance. We next compared the magnitudes of the travel time perturbation to the estimated change in the front due its movement. We concluded 
that since the front could move upwards of $10 \mathrm{~km}$ during a tidal cycle, which would result in a travel time perturbation of up to 49 msecs, that the travel time perturbations due to the internal tide and internal wave fields would be a small, second order effect.

Whereas in this thesis we have used Fourier analysis techniques to extract the internal tide and internal wave amplitudes, there exist several other methods which could be employed with varying degrees of success. One method is the Lagrangian contour following method used by Wang et al. [1991] that traces the minimum temperature through vertically interpolated temperature records. Another method is to determine the vertical velocities using the principle of the conservation of temperature which Holloway [1984] gives as

$$
w=-\frac{\partial T / \partial t}{\partial T / \partial z}
$$

Both methods are more suited to situations where there are several temperature records in the vertical and where a minimal amount of smoothing needs to be done to remove the "spiking" due to eddies and other oceanographic processes. There also exist more sophisticated methods of tidal harmonic analysis which can be explored to extract the amplitudes such as those used by Foreman [1977] (cited in Holloway, 1984). Finally, a last method to estimate the height of the $M 2$ internal tide would be to calculate the movement of the thermocline using the anchor station CTD casts at the SW array.

The analysis was hindered by our ignorance of the direction of propagation of the internal wave field. Levine and Richman [1989] have devised a method, whereby using a single mooring of current meters and thermistors, the direction could be resolved. Perhaps such an analysis of both the current meter and the temperature information may result in resolving the propagation direction. This information would allow us to check our calculations versus the measured data with much less uncertainty.

Much more work remains to be done in fully understanding the nature of the internal tide and internal wave fields. The internal wave spectrum could be compared 
to the Garrett-Munk internal wave model. The travel time perturbation covariance was computed for the $\theta=0$ and $\theta=90$ cases, but could also be computed for the isotropic case. Finally, future work in the Barents Sea could be done to resolve the phase relationship of the the displacement of the BSPF to the barotropic tide and to the internal tide, thereby allowing a more accurate determination of the acoustic signal travel time perturbation. 


\section{Bibliography}

Apel, J. R., Principles of Ocean Physics, Academic Press, 1987.

Baines, P. G., The generation of internal tides over steep continental slopes, Phil. Trans. Roy. Soc. London, Ser. A, 277, 27-58, 1974.

Baines, P. G., Internal tides, internal waves, and near-inertial motions, In: Baroclinic Processes on Continental Shelves, edited by C. N. K. Mooers, American Geophysical Union, Washington D. C., 1986.

Brekhovskikh, L.M. and Y.P. Lysanov, Fundamentals of Ocean Acoustics, SpringerVerlag, 1991.

Chereskin, T. K., Generation of internal waves in Massachusetts Bay, J. Geophys. Res., 88, 2649-2661, 1983.

Curtin, T. B. and C. N. K. Mooers, Observation and interpretation of a high frequency internal wave packet and surface slick pattern, J. Geophys. Res., 80, 882-894, 1985.

Essen, H. H., F. Schirmer, and S. Sirkes, Acoustic remote sensing of internal waves in shallow water, J. Remote Sensing, 4, 33-47, 1983.

Farmer, D. M. and J. D. Smith, Tidal interaction of stratified flow with a sill in Knight Inlet, Deep-Sea Res., 27A, 239-254, 1980.

Foreman, M. G. G., Manual for tidal heights analysis and prediction, Pac. Mar. Sci. Rep., 77-10, pp. 101, 1977.

Gargett, A. E., Generation of internal waves in the Strait of Georgia, British Columbia, Deep-Sea Res., 23, 17-32, 1976.

Garrett, C. J. R. and W. H. Munk, Space-time scales of internal waves, Geophys. Fluid Dyn., 2, 225-264, 1972.

Gill, A. E. Atmosphere-Ocean Dynamics, Academic Press, 1982.

Gjevik, B., Model Simulations of tides and shelf waves along the shelves of the Norwegian-Greenland-Barents sea, In, Modelling Marine Systems, vol. 1, ed A. M. Davies, CRC Press Inc., 1990.

Jin, G., Internal waves perturbation formulation, unpublished manuscript, pp. 4, 1992.

Halpern, D., Observations on short period internal waves in Massachusetts Bay, $J$. Mar. Res., 29, 116-132, 1971. 
Hamilton, G. R., Time variations of sound speed over long paths in the ocean, In: International workshop on low-frequency propagation and noise, Woods Hole, Massachusetts, Oct. 14-19, 1974, 7-30, 1977.

Holloway, P. E., On the semidiurnal tide at a shelf-break region on the Australian north west shelf, J. Phys. Oceanogr., 1787-1799, 1984.

Hopkins, T. S., The GIN Sea-A synthesis of its physical oceanography and literature review 1972-1985, Earth-Science Rev., 30, 175-318, 1991.

Howe, B. M., P. F. Worcester, and R. C. Spindel, Ocean acoustic tomography: Mesoscale velocity, J. Geophys. Res., 92, 3785-3805, 1987

Huthnance, J. M., Large tidal currents near Bear Island and related tidal energy losses from the North Atlantic, Deep-Sea Res., 28A, 51-70, 1981.

Johannessen, O. M., Brief overview of the physical oceanography, In: The Nordic Seas, edited by B. G. Hurdle, Springer-Verlag, New York, 1986.

Käse, R. H. and R. A. Clarke, High frequency internal waves in the upper thermocline during GATE, Deep-Sea Res., 25, 815-825, 1978.

Käse, R. H. and G. Siedler, Internal wave kinematics in the upper tropical Atlantic, Deep-Sea Res., 26, Supp. I, 161-189, 1980.

LeBlond, P. H. and L. A. Mysak, Waves in the Ocean, Elsevier Scientific Publishing Co., Amsterdam, 1978.

Levine, M. D. and J. G. Richman, Extracting the internal tide from data: methods and observations from the Mixed Layer Dynamics Experiment, J. Geophys. Res., 94, 8125-8134, 1989.

Lynch, J. F., S. D. Rajan, and G. V. Frisk, A comparison of broadband and narrowband modal inversions for bottom geoacoustic properties at a site near Corpus Christi, Texas, J. Acoust. Soc. Am., 89, 648-665, 1991.

Maxworthy, T., A note on the internal solitary waves produced by tidal flow over a three-dimensional ridge, J. Geophys. Res., 84, 338-346, 1979.

Müller, P. and D. J. Olbers, On the dynamics of internal waves in the deep ocean, J. Geophys. Res., 80, 3848-3860.

Munk, W. H. and C. Wunsch, Ocean acoustic tomography: A scheme for large scale monitoring, Deep-Sea Res., 26A,123-161,1979.

Olbers, D. J., Models of the oceanic internal wave field, Rev. Geophys. Space Phys., $21,1567-1606$.

Pawlowicz, R., Preliminary data report for the Barents Sea polar front experiment part 1: CTD surveys, unpublished manuscript, pp. 56, 1992. 
Pierce, A. D., Extension of the method of normal modes to sound propagation in an almost-stratified medium, J. Acoust. Soc. Am., 37, 19-27, 1965.

Rajan, S. D., J. F. Lynch, and G. V. Frisk, Perturbative inversion methods for obtaining bottom geoacoustic parameters in shallow water, J. Acoust. Soc. Am., 82, 998-1017, 1987.

Rattray, M., Jr., J. G. Dworski, and P. E. Kovala, Generation of long internal waves at the continental slope, Deep-Sea Res., Suppl. to 16, 179-195, 1969.

Sawyer, C., Tidal phase of internal wave generation, J. Geophys. Res., 88, 2642$2648,1983$.

Schwiderski, E. W., Tides, In, The Nordic Seas, edited by B. G. Hurdle, SpringerVerlag, New York, pp. 191-209, 1986.

Shang, E. C., Ocean acoustic tomography based on adiabatic mode theory, J. Acoust. Soc. Am., 85, 1531-1537, 1989.

Steinberg, J. C. and T. G. Birdsall, Underwater sound propagation in the Straits of Florida, J. Acoust. Soc. Am., 39, 301-315, 1966.

Thorpe, S. A., The excitation, dissipation, and interaction of internal waves in the deep ocean, J. Geophys. Res., 80, 328-338, 1975.

von der Heydt, K., J. Kemp, and J. F. Lynch, Array data acquisition with wireless LAN telemetry as applied to shallow water tomography in the Barents Sea, Woods Hole Oceanog. Inst. Tech. Rept., WHOI-92-44, pp. 39, 1992.

Wang, J., R. G. Ingram, and L. A. Mysak, Variability of internal tides in the Laurentian Channel, J. Geophys. Res., 96, 16859-16875, 1991.

Worcester, P. F., Reciprocal acoustic transmission in a midocean environment, $J$. Acoust. Soc. Am., 62, 895-905, 1977.

Wunsch, C., Internal tides in the ocean, Rev. Geophys., 13, 167-182, 1975. 